ARTICLE

\title{
The RNase MCPIP3 promotes skin inflammation by orchestrating myeloid cytokine response
}

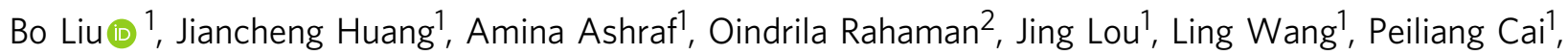

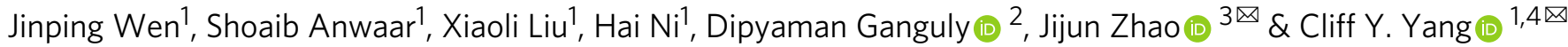

$\mathrm{CCCH}$ zinc finger proteins resolve immune responses by degrading the mRNAs of inflammatory cytokines such as tumor necrosis factor (TNF) and interleukin (IL)-6. Here we report that one such family member, monocyte chemotactic protein-induced protein 3 (MCPIP3, also named $\mathrm{ZC} 3 \mathrm{H} 12 \mathrm{C}$ or Regnase-3), promotes skin inflammation by simultaneously enhancing TNF in macrophages and repressing IL-6 in plasmacytoid dendritic cells (pDCs). MCPIP3 is positively associated with psoriasis pathogenesis, and highly expressed by macrophages and pDCs. MCPIP3-deficient macrophages produce less TNF and IL-12p40. However, MCPIP3-deficient pDCs secrete significantly more IL-6. This enhanced intradermal IL-6 may alleviate imiquimod-induced skin inflammation. As a result, MCPIP3-deficient mice are protected from imiquimod-induced psoriasiform lesions. Furthermore, early exposure to pDC-derived IL-6 suppresses macrophage-derived TNF and IL-12p40. Mechanistically,

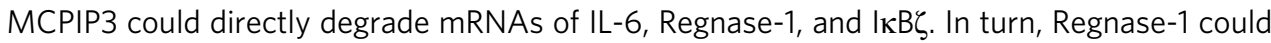
degrade MCPIP3 mRNAs. Our study identifies a critical post-transcriptional mechanism that synchronizes myeloid cytokine secretion to initiate autoimmune skin inflammation.

\footnotetext{
${ }^{1}$ Department of Immunology, Sun Yat-sen University, Zhongshan School of Medicine, Guangzhou, Guangdong, China. ${ }^{2}$ IICB-Translational Research Unit of Excellence, CSIR-Indian Institute of Chemical Biology, Kolkata, India. ${ }^{3}$ Department of Rheumatology and Immunology, The First Affiliated Hospital, Sun Yatsen University, Guangzhou, China. ${ }^{4}$ Key Laboratory of Tropical Disease Control (Sun Yat-Sen University), Ministry of Education, Guangzhou, China.

凶email: zhjij@mail.sysu.edu.cn; yangkeli6@mail.sysu.edu.cn
} 
delicate balance between pro-inflammatory and antiinflammatory immune responses is required to clear pathogens without causing autoimmunity ${ }^{1}$. Tumor necrosis factor (TNF) and interleukin (IL)-6 are potent inflammatory cytokines that regulate immunity and disease pathogenesis ${ }^{2}$. Aberrant overproduction of TNF had been implicated in the pathogenesis of chronic inflammatory diseases such as rheumatic arthritis, psoriatic lesions/arthritis, and Crohn's disease ${ }^{3,4}$. Similarly, elevated IL-6 expression led to idiopathic juvenile arthritis and other chronic inflammations ${ }^{2,5}$. While TNFtargeted blockade had been shown to be effective in treating psoriasis and other autoimmune diseases, IL-6-targeted blockade had not been equally successful ${ }^{6}$. Whereas the transcriptional control of TNF and IL- 6 has been extensively investigated, the post-transcriptional regulation of TNF/IL-6 is less understood.

RNA-binding proteins are major components of regulatory feedback loops that maintain immune tolerance and limit inflammation ${ }^{7}$. Cys-Cys-Cys-His (CCCH) zinc finger proteins are a subclass of RNA-binding proteins that regulate mRNA splicing, polyadenylation, export, translation, and decay $^{8}$. Although the physiological functions of most $\mathrm{CCCH}$ zinc finger proteins are still uncertain, recent reports suggest that the tristetraprolin (TTP), roquin, and monocyte chemotactic protein-induced protein (MCPIP) families, are critical negative regulators of inflammatory responses $^{9-11}$. TTP, roquin 1 , and Regnase-1 (also known as MCPIP1; encoded by $Z c 3 h 12 a$ ) belong to an intricate mRNA degradation network that maintains immune homeostasis by repressing inflammatory cytokines such as IL-6 and TNF in macrophages $^{12-15}$. Besides macrophages, roquin 1 and Regnase- 1 suppress the activation, differentiation, and IL-17A production of $\mathrm{CD}^{+}$helper T cells ${ }^{16-20}$. TTP, roquin 1 and Regnase- 1 could be evolutionarily closely related, as their genetically deficient mouse models suffer from a multitude of severe inflammatory autoimmune diseases ${ }^{10,15,21,22}$. By comparison, other MCPIP members appear to have similar but less significant roles than Regnase-1. MCPIP4 (also named TFL; encoded by $Z c 3 h 12 d$ )-deficient mice were healthy but exhibited excessive T-cell activation in a multiple sclerosis model ${ }^{23}$. MCPIP3 (also named Regnase-3; encoded by $Z c 3 h 12 c)$-deficient mice were also healthy, despite some lymphadenopathy and an increase in serum interferon (IFN)- $\gamma^{24}$. Thus far, all characterized $\mathrm{CCCH}$ zinc finger proteins have been reported to be repressors of cytokine production and immune cell activation.

In humans, single nucleotide polymorphisms (SNPs) in MCPIP3 were identified in genome-wide association studies (GWAS) to be highly associated with psoriasis ${ }^{25}$. Psoriasis is a common chronic inflammatory skin disease with a prevalence of an estimated $1-3 \%$ of the worldwide population ${ }^{26}$. Heavy dermal infiltration of immune cells, such as myeloid cells and T cells, is critical for epidermal thickening ${ }^{27}$. Subsequently, TNF and IL-23 produced by myeloid cells drive the production of IL-17A by $\mathrm{T}$ cells ${ }^{28}$. Clinical studies and targeted therapies have demonstrated that the cytokines TNF, IL-23, and IL-17A have pivotal roles in psoriasis pathogenesis ${ }^{29}$. Thus, the exact role of MCPIP3 in psoriasis warrants further investigation.

In this report, we identify MCPIP3 as a critical regulator of inflammatory cytokines during early phase of psoriasis pathogenesis. We show that MCPIP3 promotes TNF production in macrophages, and represses IL-6 secretion in pDCs. We demonstrate that MCPIP3 degrades multiple targets, such as Il6, $Z c 3 h 12 a$, and Nfkbiz mRNAs, to achieve this differential cytokine regulation.

\section{Results}

MCPIP3 is positively associated with psoriasis pathogenesis. A previously published GWAS study identified one SNP in
$\mathrm{ZC} 3 \mathrm{H} 12 \mathrm{C}$ (the gene encoding MCPIP3) that is highly associated with psoriasis ${ }^{25}$. This SNP (rs4561177) is located at $\sim 1.8 \mathrm{~kb}$ upstream from the $\mathrm{ZC} 3 \mathrm{H} 12 \mathrm{C}$ transcriptional start site (Fig. 1a). The bioinformatical database Genotype-Tissue Expression (GTEx, www.gtexportal.org) suggested that this particular SNP could be an expression quantitative trait locus (eQTL) that controls human gene transcription (Supplementary Fig. 1a). To see if this SNP affects ZC3H12C expression, we cloned $\sim 1 \mathrm{~kb}$ of upstream promoter region including the SNP into a luciferase reporter system. We found that this single $A$ to $G$ conversion increased luciferase reporter expression by $\sim 50 \%$ when compared to the same promoter region with no SNP (Fig. 1b). In order to determine if this increased MCPIP3 expression is correlated with psoriasis, we analyzed human $Z C 3 H 12 C$ transcripts from psor-

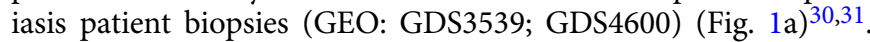
We found that $\mathrm{ZC} 3 \mathrm{H} 12 \mathrm{C}$ transcripts were significantly higher in lesional skin than non-lesional skin from the same psoriasis patients (Fig. 1c). Consistently, we found that $\mathrm{ZC} 3 \mathrm{H} 12 \mathrm{C}$ expression is normalized after successful clinical treatment of brodalumab, an anti-IL-17A neutralizing antibody (GEO: GSE53552) (Fig. 1d $)^{32}$. Here, we showed that MCPIP3 expression is positively associated with psoriasiform lesions.

Next, we examined MCPIP3 mRNA transcripts in human and murine immune cells. From human peripheral blood mononuclear cells (PBMCs), we observed that ZC3H12C mRNA was highly expressed in a broad group of myeloid cells, including pDCs, CD1C ${ }^{+}$DCs, monocytes and monocyte-derived macrophages (MoMФs) (Fig. 1e). Human and murine MCPIP3 are highly conserved, especially at the key functional NYN nuclease and $\mathrm{CCCH}$ RNA-binding domains $(100 \%$ conserved at the protein level) (Supplementary Fig. 1b). In naïve murine immune populations, $Z c 3 h 12 c$ expression was the highest in plasmacytoid dendritic cells (pDCs) (Fig. 1f). This pattern is consistent with RNA-Seq data from the Immunological Genome Project (IMMGEN, www.immgen.org) (Supplementary Fig. 1c). In pDCs, $Z c 3 h 12 c$ mRNA expression was further increased after TLR7/8 agonist R848 stimulation, with a peak around $16 \mathrm{~h}$ (Fig. 1g). Interestingly, $Z c 3 h 12 c$ expression in pDCs was unchanged after TLR9 agonist CpG A activation (Fig. 1g). In contrast to CpG A, CpG B activates TLR9 in the late endosome and upregulates NFkB dependent cytokines IL-6 and TNF much more than CpG $\mathrm{A}^{33}$. We found that CpG B could upregulate $Z c 3 h 12 c$ expression in pDCs, albeit to a lesser extent than R848 (Fig. 1g). Here we show that MCPIP3 expression is enriched in naïve and activated pDCs.

Unstimulated murine macrophages expressed relatively few MCPIP3 transcripts (Fig. 1f). After R848-activation, Zc3h12c transcripts was upregulated by $>6$-fold in bone marrow-derived $\mathrm{CD}_{11 \mathrm{~b}}{ }^{+} \mathrm{F} 4 / 80^{+}$macrophages (BMDMs) and by $>3$-fold in peritoneal cavity $\mathrm{CD}_{11 \mathrm{~b}}{ }^{+} \mathrm{F} 4 / 80^{+}$macrophages (Per. MФs) (Fig. 1f). Still, $Z c 3 h 12 c$ mRNA in unstimulated pDCs was $\sim 1$ fold higher than that of R848-activated macrophages (Fig. 1f). This meant that $Z c 3 h 12 c$ expression in R848-activated pDCs was $\sim 14$-fold higher than that of activated macrophages (Fig. 1g). Next, we carefully monitored the kinetics of MCPIP3 expression in activated macrophages. $Z c 3 h 12 c$ mRNA expression peaked at $4 \mathrm{~h}$ after R848 activation, but remained quite low at later timepoints (Fig. 1h). Here we show that MCPIP3 is transiently expressed in early activated macrophages.

To study the function of MCPIP3 in pDCs and macrophages, we generated germline genetic knockout mice $\left(Z c 3 h 12 c^{-1-}\right)$ and conditional knockout mice $\left(Z c 3 h 12 c^{\mathrm{ll} / \mathrm{fl}}\right)$ with $L y z 2-C r e\left(Z c 3 h 12 c^{\mathrm{M} \Phi \Delta}\right)$ and Itgax-Cre $\left(Z c 3 h 12 c^{\mathrm{DC} \Delta}\right)$ (Supplementary Fig. $\left.1 \mathrm{~d}-\mathrm{i}\right)^{34,35}$. We observed low occurrence of lymphadenopathy in $>12$ weeks old $Z c 3 h 12 c^{-1-}$ and $Z c 3 h 12 c^{\mathrm{M} \Phi \Delta}$ mice, albeit this difference was not statistically significant (Supplementary Fig. 1j). The penetrance and 
a

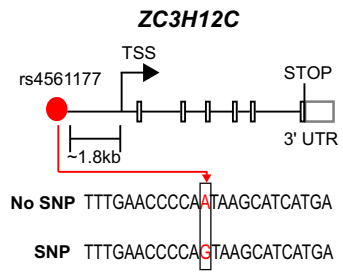

b

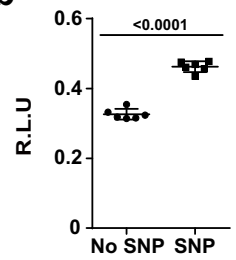

$\mathbf{C}$

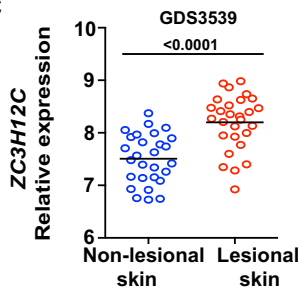

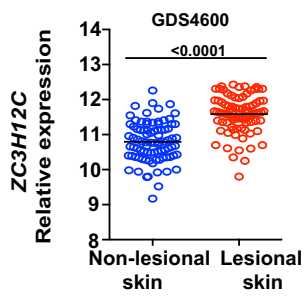

e

d

GSE53552
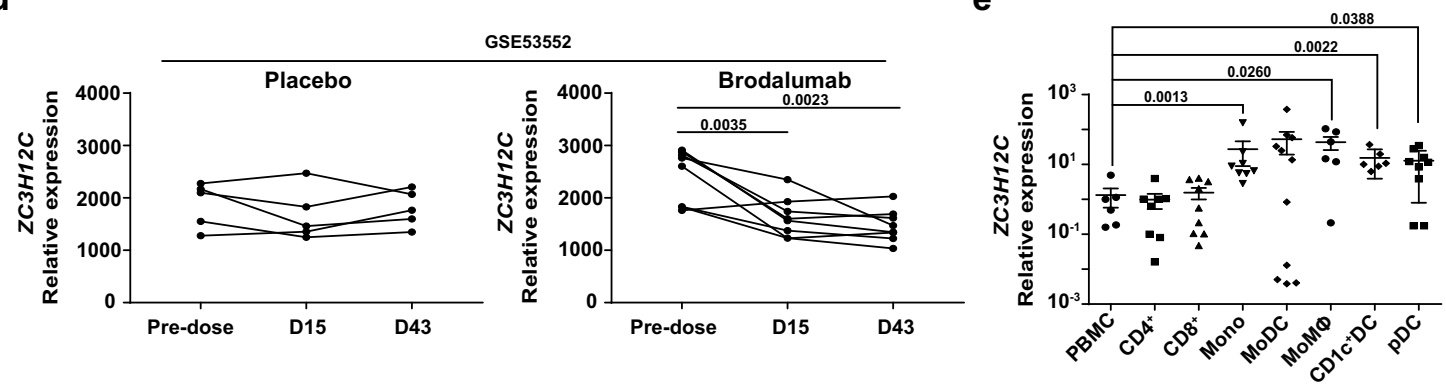

f

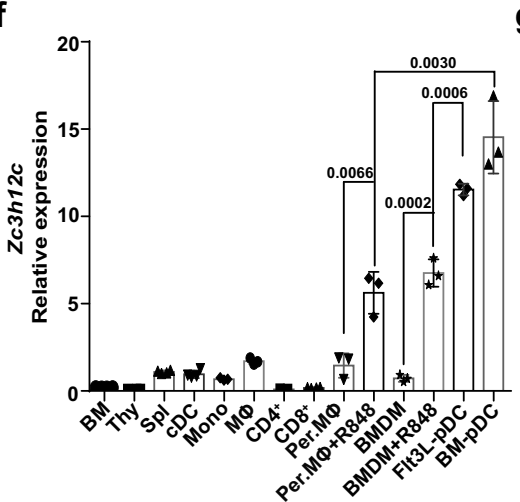

g

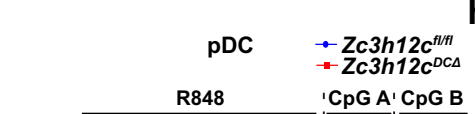

h

$-Z c 3 h 12 c^{+/+}$
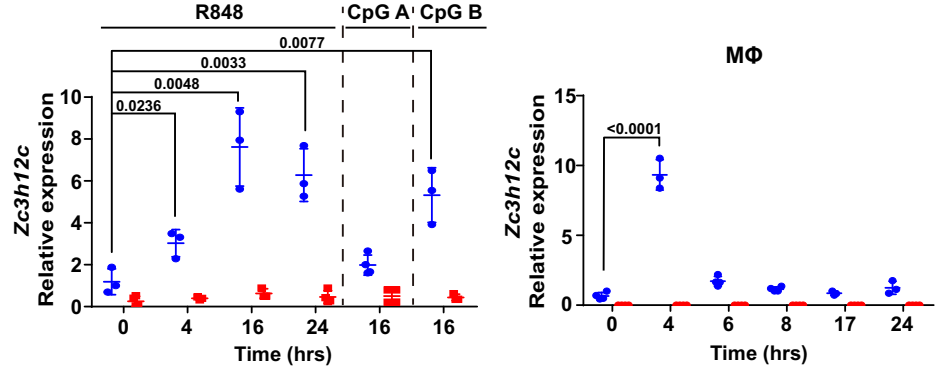

Fig. 1 MCPIP3 is positively associated with psoriasis pathogenesis. a Schematics indicating the location of SNP rs4561177 in human ZC3H12C.

b Regulation of ZC3H12C expression by SNP rs 4561177 was measured by a luciferase reporter system. The promoter region ( $1 \mathrm{~kb})$ containing the SNP was inserted upstream of the luciferase gene, and then transfected into 293 T cells for assessment of reporter activity (R.L.U) ( $n=6$ replicates). c ZC $3 H 12 C$ expression in psoriatic lesion (red) and non-lesions biopsies (blue) from psoriasis patients were analyzed ( $n=28$ individuals in GDS3539; $n=85$ individuals in GDS4600). d ZC3H12C expression in psoriatic lesions after brodalumab treatment (GEO: GSE53552) was analyzed ( $n=5$ individuals for placebo, 8 individuals for brodalumab). e ZC3H12C expression in human peripheral blood mononuclear cells (PBMC) immune populations was measured by qPCR ( $n=6$ individuals for PMBC; $n=11$ individuals for other groups). $\mathbf{f} Z \mathrm{c} 3 h 12 c$ expression in murine WT immune populations was measured by qPCR ( $n=3$ mice per group). $\mathbf{g} Z c 3 h 12 c$ expression in sorted $Z c 3 h 12 c \mathrm{fl} / \mathrm{fl}$ (blue) /Zc3h12c DCA (red) BM plasmacytoid dendritic cells (pDCs) was measured by

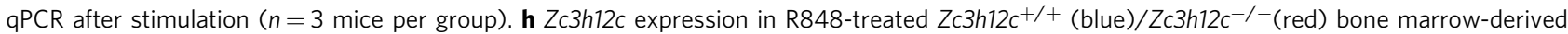
macrophages (BMDMs) was measured by GPCR at indicated timepoints ( $n=3$ mice per group). Data are representative at least three independent experiments (mean \pm S.D.; two-tailed Student's $t$ test for murine samples; two-tailed Wilcoxon matched-pair signed rank test for human samples). Source data are provided as a Source Data file.

severity of lymphadenopathy in our MCPIP3-deficient mice was much lower than previously reported 24 . For all our in vivo experiments, we utilized only $6-10$ weeks old mice, which exhibited no signs of lymphadenopathy. In general, $Z c 3 h 12 c$-deficient mice were born at normal Mendelian ratios, had normal immune development, and appeared healthy for at least 1.5 years (Supplementary Fig. 2a). Most importantly, macrophage and DC populations in $Z c 3 h 12 c^{-1-} / Z c 3 h 12 c^{\mathrm{M} \Phi \Delta} / Z c 3 h 12 c^{\mathrm{DC} \Delta}$ mice are normal compared to littermate controls (Supplementary Fig. 2b-i). Thus, we conclude that MCPIP3 is dispensable for macrophage or DC development.

Early TNF production is impaired in MCPIP3-deficient macrophages. Since MCPIP3 was highly expressed in activated macrophages, we investigated the role of MCPIP3 in macrophage function. We found a significant reduction of $\operatorname{Tnf}$ transcripts or TNF protein in $Z c 3 h 12 c^{-1-}$ BMDMs at early phase $(0-4 \mathrm{~h})$ but not at late phase (6-24h) after activation (Fig. 2a, b, Supplementary
Fig. 3a, b). In contrast, IL-6 production was unaffected at all timepoints by MCPIP3-deficiency, though it was little secreted at early phase (Fig. 2c, Supplementary Fig. 3c). Using TNF intracellular staining, we have observed similar TNF phenotype when comparing $Z c 3 h 12 c^{+/+} / Z c 3 h 12 c^{-/-}$or $Z c 3 h 12 c^{\mathrm{fl} / \mathrm{fl}} / Z c 3 h 12 c^{\mathrm{M} \Phi \Delta}$ macrophages at early phase (Fig. 2d, e). In addition to TLR7 agonists, this early TNF secretion defect was also seen in MCPIP3deficient macrophages activated by various TLR1/2/3/4 ligands (Fig. 2d, e). Besides BMDMs, we also examined MCPIP3-deficient Per. MФs, and found a similar defect in early TNF production with R848 and Pam3CSK4 treatment (Fig. 2f). Besides TNF, IL-12 is a key inflammatory cytokine reported to be involved in early psoriasis pathogenesis ${ }^{36}$. We found that $I l 12 p 40$ transcripts were also decreased in R848-activated MCPIP3-deficient macrophages (Fig. 2g). Using intracellular staining, we observed $\sim 50 \%$ reduction of IL-12p40 production by macrophages after R848, Pam3CSK4, or LPS activation (Fig. 2h, i). Although serum IFN- $\gamma$ was reported to be affected by MCPIP3, we saw little IFN- $\gamma$ production by either 
a
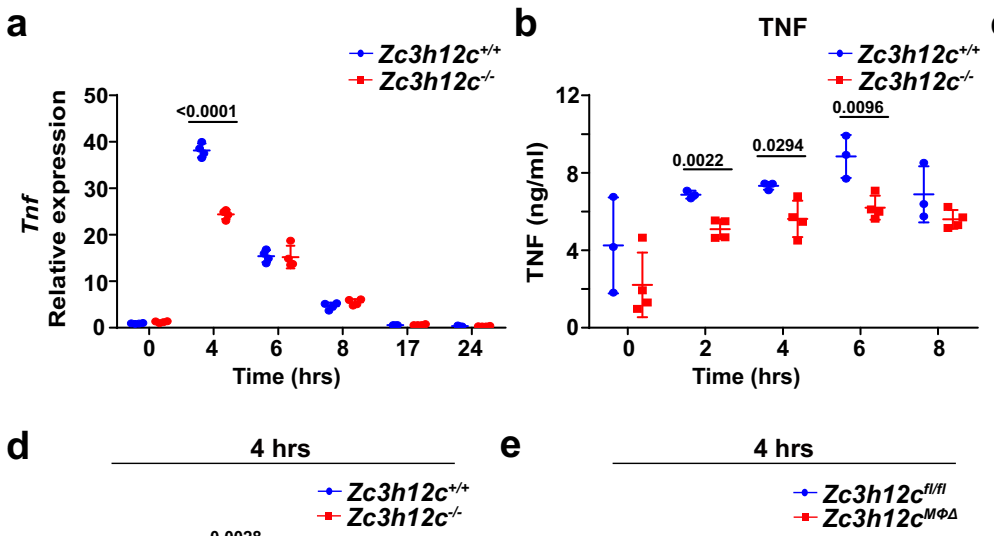

e
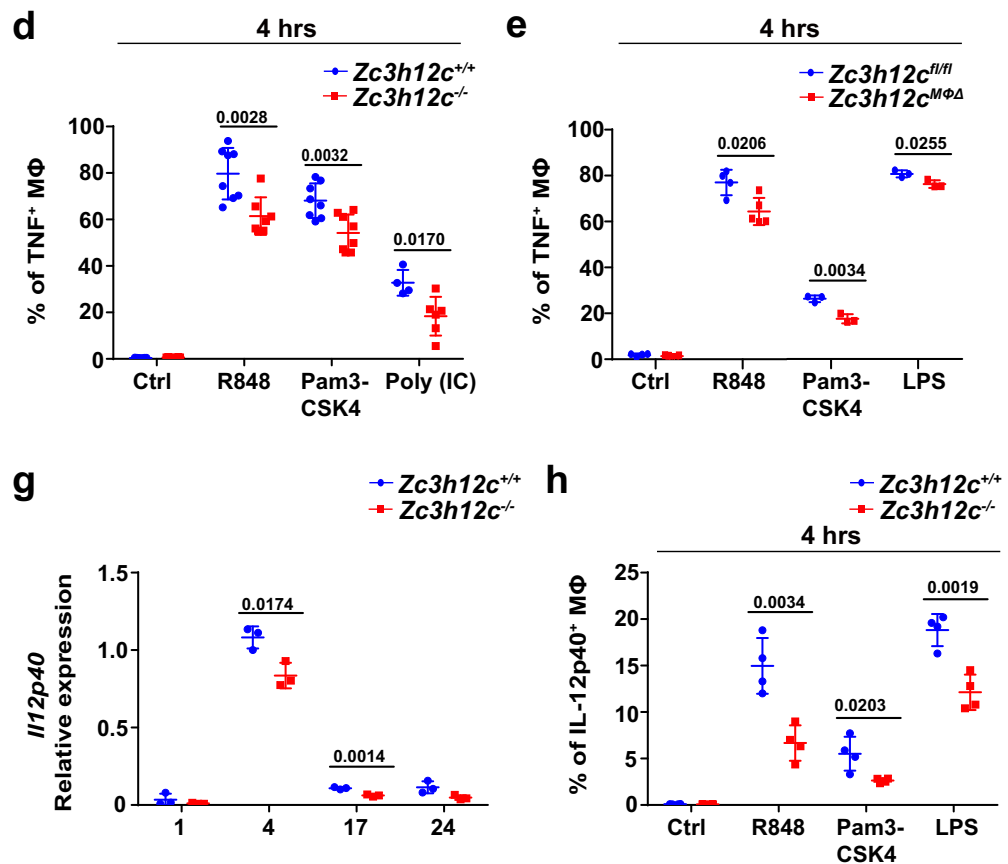
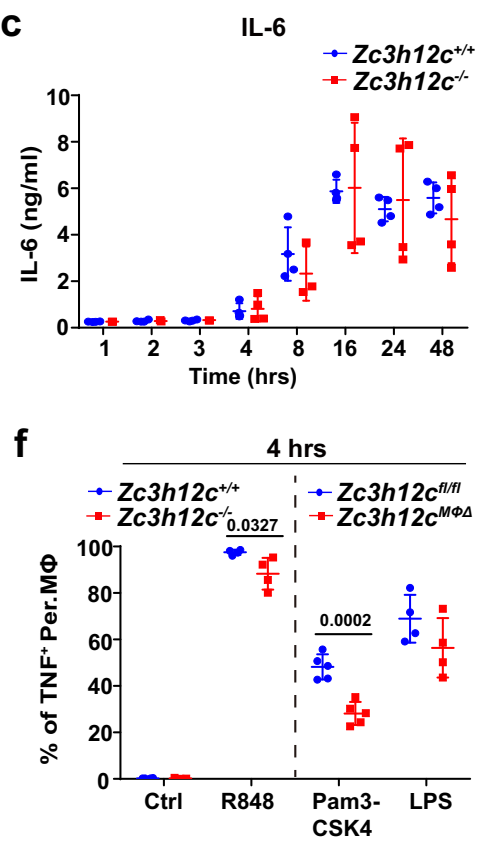

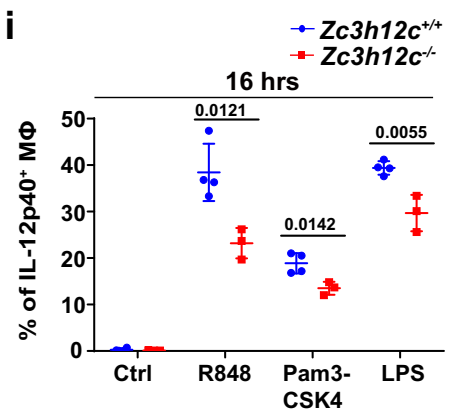

Fig. 2 Early TNF production is impaired in MCPIP3-deficient macrophages. a Tnf expression in R848-treated $Z c 3 h 12 c^{+/+}$(blue)/Zc3h12c $c^{-/-}($red) bone marrow-derived macrophages (BMDMs) was measured by qPCR at indicated timepoints ( $n=3$ mice per group). b, c TNF(b) and IL-6(c) secretion by R848-treated $Z c 3 h 12 c^{+/+} / Z c 3 h 12 c^{-/}$BMDMs was measured by ELISA at indicated timepoints $\left(n=3\right.$ mice for $Z c 3 h 12 c^{+/+} ; n=4$ mice for $Z c 3 h 12 c^{-/-}$). $\mathbf{d}-\mathbf{f}$ TNF production by

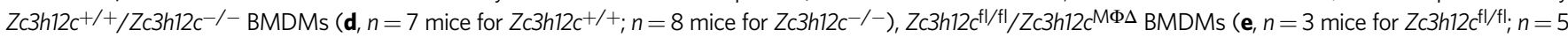
mice for $Z c 3 h 12 c^{\mathrm{M} \Phi \Delta}$ ), and purified $Z c 3 h 12 c^{+/+} / Z c 3 h 12 c^{-/-}$or $Z c 3 h 12 c^{f l / f l} / Z c 3 h 12 c^{\mathrm{M} \Phi \Delta}$ peritoneal macrophages (Per.M $\left.\mathrm{M}\right)\left(\mathbf{f}, n=3 \mathrm{mice}\right.$ for $Z c 3 h 12 c^{f l} / f l ; n=5$ mice for $Z c 3 h 12 c^{M \Phi \Delta}$ ) was measured by intracellular staining after $4 \mathrm{~h}$ of stimulation with indicated ligands. $\mathbf{g} / 12 \mathrm{p} 40$ expression in R848-treated $Z \mathrm{c} 3 \mathrm{~h} 12 \mathrm{c}^{+} / \mathrm{t} / \mathrm{Zc} 3 \mathrm{~h} 12 \mathrm{c}^{-/-}$ BMDMs was measured by qPCR at various timepoints ( $n=3$ mice per group). $\mathbf{h}, \mathbf{i} \mathrm{IL}-12 \mathrm{p} 40$ production by $Z \mathrm{c} 3 \mathrm{~h} 12 \mathrm{c}^{+/} / \mathrm{l} / \mathrm{Zc} 3 \mathrm{~h} 12 \mathrm{c}^{-/-} \mathrm{BMDMs}$ was measured by intracellular staining after $4 \mathrm{~h}\left(\mathbf{h}, n=4\right.$ mice per group) or $16 \mathrm{~h}\left(\mathbf{I}, n=4\right.$ mice for $Z c 3 h 12 c^{+/ t} ; n=3$ mice for $Z c 3 h 12 c^{-/-)}$stimulation with indicated ligands. Data are representative of eight $(\mathbf{d}, \mathbf{e})$, four $(\mathbf{a}, \mathbf{b})$, three $(\mathbf{h}, \mathbf{i})$, two $(\mathbf{c}, \mathbf{f}, \mathbf{g})$ independent experiments (mean \pm S.D.; two-tailed Student's $t$ test). Source data are provided as a Source Data file.

$Z c 3 h 12 c^{+/+}$or $Z c 3 h 12 c^{-/-}$BMDMs (Supplementary Fig. $\left.3 \mathrm{~d}\right)^{24}$. Thus, we conclude that MCPIP3 promotes TNF and IL-12p40 production in macrophages.

Macrophages balance diverse functions, such as their proinflammatory function and wound repair, by polarizing into M1 or M2 macrophages under different stimuli, such as LPS or IL- ${ }^{37}$. To see if MCPIP3 affects M1/M2 polarization and associated functions, we subjected $Z c 3 h 12 c^{+/+}$and $Z c 3 h 12 c^{-1-}$ BMDMs to LPS and IL-4 treatments in vitro, and measured several key macrophage polarization genes by qPCR. Judging by the signature genes such as Arg1 and Nos2, the effects of R848 stimulation were more similar to those of LPS (Supplementary Fig. 3e). Similar to R848 treatment, $Z c 3 h 12 c$ expression was upregulated in LPStreated, but not in IL-4-treated BMDMs (Supplementary Fig. 3f). $\mathrm{ZC} 3 \mathrm{H} 12 \mathrm{C}$ expression was also higher in M1-conditioned human peripheral macrophages from PBMCs (Supplementary Fig. 3g). However, expression levels of Arg1 and Nos2 in remained unchanged in R848-treated MCPIP3-deficient BMDMs
(Supplementary Fig. 3h). Instead, MCPIP3-deficient BMDMs expressed more Ilr4a transcripts and fewer Ifnar1/Ifnar2 transcripts, which are signatures associated with alternatively activated macrophages $^{38}$ (Supplementary Fig. 3h). Because TNF from M1polarized macrophages is crucial for septic shock, we induced

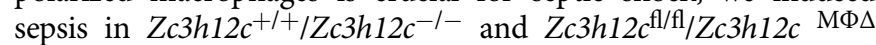
mice with LPS. We found that all mice had the same survival rate (Supplementary Fig. 3i). We also infected $Z c 3 h 12 c^{+/+} / Z c 3 h 12 c^{-1-}$ mice with Listeria monocytogenes, and found no differences in liver or spleen bacterial load (Supplementary Fig. 3j). Since M2 macrophages participate in tissue repair, we inflicted skin wounds on $Z c 3 h 12 c^{+l+} / Z c 3 h 12 c^{-1-}$ mice and found no difference in wound repair between them (Supplementary Fig. 3k). Thus, MCPIP3 is dispensable for classic M1/M2 macrophage functions.

IL-6 production is enhanced in MCPIP3-deficient pDCs. Next, we examined the role of MCPIP3 in activated pDCs. The most well-known ability of pDCs is its massive production of IFN- $\alpha$, 
a

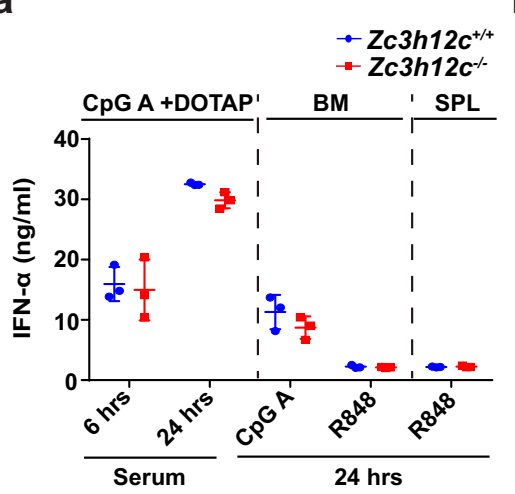

d

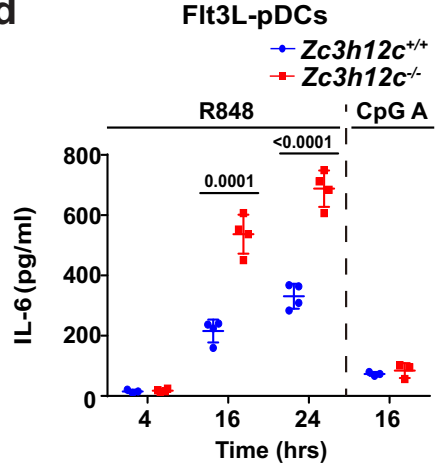

b

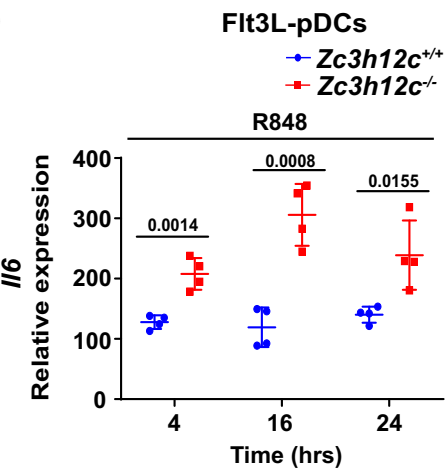

C
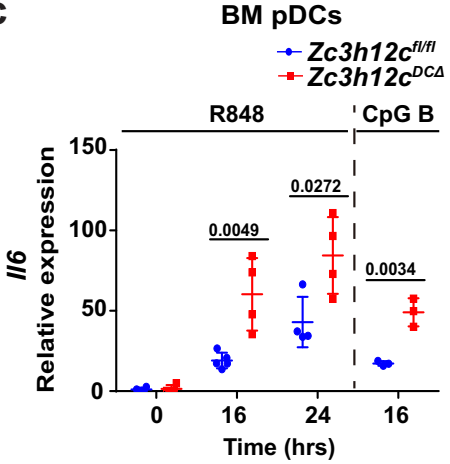

e

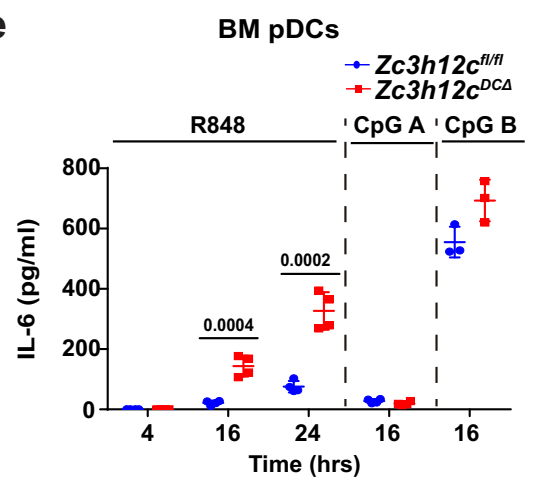

f
SPL CDCs

$\rightarrow Z c 3 h 12 c^{+/+}$

$-Z c 3 h 12 c^{-1-}$

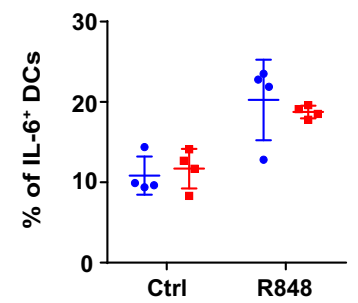

g

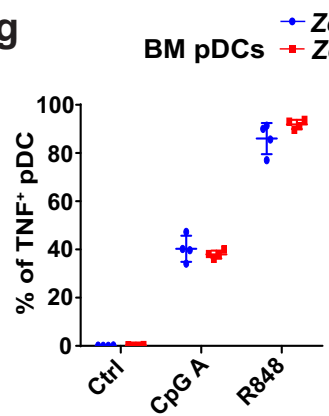

$h$
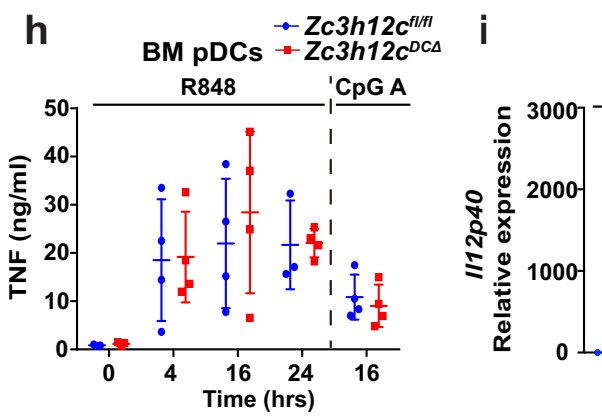

FIt3L-pDC $\quad \stackrel{-Z c 3 h 12 c^{+/+}}{\rightarrow Z c 3 h 12 c^{-/}}$

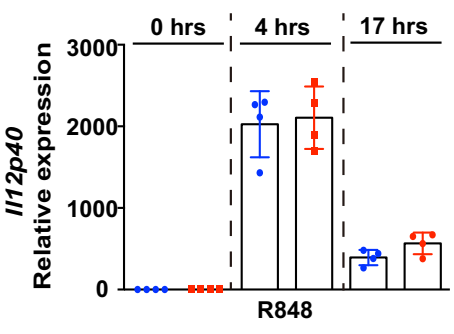

Fig. 3 IL-6 production is enhanced in MCPIP3-deficient pDCs. a IFN- $\alpha$ secretion by $Z c 3 h 12 c^{+/+}$(blue)/Zc3h12c $c^{-/-}$(red) plasmacytoid dendritic cells (pDCs) was measured by ELISA after stimulation ( $n=3$ mice per group). b $1 / 6$ expression in $Z c 3 h 12 c^{+/} / / 2 c 3 h 12 c^{-/}-$FL3TL-pDCs was measured by qPCR ( $n=4$ mice per group). $\mathbf{c} / 16$ expression in sorted $Z c 3 h 12 c \mathrm{fl} / \mathrm{fl} / Z \mathrm{Zc} 3 \mathrm{~h} 12 c^{\mathrm{DC} \Delta}$ bone marrow (BM) pDCs was measured by qPCR ( $n=4$ mice per group). $\mathbf{d} \mid \mathrm{LL}-6$

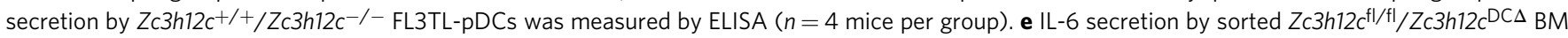

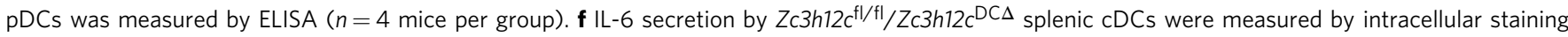
after R848 activation ( $n=4$ mice per group). $\mathbf{g}$ TNF production by $Z c 3 h 12 c^{+/+} / Z c 3 h 12 c^{-/-}$BM pDCs was measured by intracellular staining after $4 \mathrm{~h}$ of stimulation ( $n=4$ mice per group). $\mathbf{h}$ TNF secretion by sorted $Z c 3 h 12 c^{\mathrm{fl} / \mathrm{fl} /} Z \mathrm{Zc} 3 \mathrm{~h} 12 c^{\mathrm{DC} \Delta} \mathrm{BM} \mathrm{pDCs}$ was measured ELISA ( $n=4$ mice per group). i $/ / 12 p 40$ expression in Zc3h12c $c^{+/+} / Z c 3 h 12 c^{-/-}$FL3TL-pDCs was measured by qPCR ( $n=4$ mice per group). Data are representative of six (a), three (b-e), or two $(\mathbf{f}-\mathbf{i})$ independent experiments (mean \pm S.D.; two-tailed Student's $t$ test). Source data are provided as a Source Data file.

especially under TLR9 agonist CpG A stimulation ${ }^{39}$. To determine if MCPIP3 regulates IFN- $\alpha$ production by pDCs, we stimulated $Z c 3 h 12 c^{+l+} / Z c 3 h 12 c^{-l-}$ or $Z c 3 h 12 c^{\mathrm{fl} / \mathrm{fl}} / Z c 3 h 12 c^{\mathrm{DC} \Delta}$ pDCs in vitro or in vitro with CpG A, CpG B, or R848, and measured their IFN- $\alpha$ production by intracellular staining and ELISA. We found that MCPIP3-deficient mice had normal serum IFN- $\alpha$ production after injection with $\mathrm{CpG} A$ associated with DOTAP in vivo (Fig. 3a). Further in vitro experiments showed that MCPIP3-deficiency did not affect IFN-a production from pDCs from bone marrow, spleen, or bone marrow-derived pDCs cultured with FL3TL (FL3TL-pDCs) (Fig. 3a, Supplementary Fig. 4e, f). MCPIP3 was also dispensable for immunity against acute and chronic LCMV viral infections ${ }^{40}$ (Supplementary
Fig. 4g). Here we show that MCPIP3 is dispensable for IFN- $\alpha$ production by pDCs.

Besides IFN-I, pDCs were also known to produce cytokines such as IL- 6 and TNF $^{39}$. Since Il6 was a well-known degradative target of the $\mathrm{CCCH}$ zinc finger family, we measured IL-6 expression in activated pDCs. We detected a consistent increase in Il6 mRNA at all timepoints in $Z c 3 h 12 c^{-/-}$FL3TL-pDCs, with a $\sim 3$-fold increase at $16 \mathrm{~h}$ (Fig. 3b). We also found similar upregulation of IL-6 mRNA when comparing $Z c 3 h 12 c^{\mathrm{fl} / \mathrm{fl} /}$ $Z c 3 h 12 c^{\mathrm{DC} \Delta}$ FACS-sorted bone marrow pDCs (Fig. 3c). Using ELISA, we found that IL-6 secretion was increased by $\sim 3$-fold in MCPIP3-deficient FL3TL-pDCs and sorted bone marrow pDCs after R848 stimulation (Fig. 3d, e). Interestingly, we found very 
little upregulation of $Z c 3 h 12 c$, Il6 mRNA or IL-6 protein, with CpG A stimulation (Figs. 1g, 3d, e). Similarly, Il6 mRNA in CpG B-treated MCPIP3-deficient pDCs was upregulated, along with a slight increase in IL-6 secretion (Fig. 3c, e). Although $\mathrm{CD}_{11 c^{+}} \mathrm{MHCII}^{+} \mathrm{cDCs}$ slightly upregulated $\mathrm{Zc} 3 \mathrm{~h} 12 \mathrm{c}$ expression upon R848 activation, MCPIP3 was dispensable for their IL-6 or TNF production (Fig. 3f, Supplementary Fig. 4h-k). Despite MCPIP3's regulation of TNF in macrophages, we saw no difference in TNF production by activated pDCs at early or late timepoints (Fig. 3g, h). IL-12p40 expression was also unaltered in MCPIP3-deficient pDCs (Fig. 3i). Here we demonstrate that MCPIP3 intrinsically represses IL-6 production in pDCs.

Enhanced intradermal IL-6 alleviates psoriasiform skin inflammation. Since inflammatory cytokines secreted by MCPIP3-deficient pDCs or macrophages were perturbed in vitro, we wish to validate these results in a relevant disease model in vivo. $\mathrm{pDCs}$ and macrophages were thought to participate in the early initiation of the psoriasis inflammatory loop ${ }^{41-43}$. However, the definitive role of pDCs in psoriasis is still under debate ${ }^{44-47}$. We found $~ 50 \%$ enrichment $Z c 3 h 12 c$ transcripts in TLR7 agonist imiquimod (IMQ)-induced psoriasiform lesions of C57BL/6J (WT) mice (Fig. 4a) ${ }^{48}$. This increase in $Z c 3 h 12 c$ mRNA at day 1 was correspondent to a massive increase in Tnf mRNA expression, a hallmark of early inflammatory loop in psoriasis (Fig. 4b). Similarly, we noticed an upregulation of $I l 6 \mathrm{mRNA}$ in IMQtreated lesions (Fig. 4c).

Next, we measured secretion of inflammatory cytokines by psoriatic lesions of $Z c 3 h 12 c^{+/+} / Z c 3 h 12 c^{-l-}$ mice. Consistent with previous in vitro results, we found that $Z c 3 h 12 c^{-/-}$skin tissue released less TNF (Fig. 4d). IL-12p40 transcripts were also reduced (Fig. 4e). To identify the source of this TNF defect, we measured TNF secretion by skin-resident myeloid cells via intracellular staining. We found that TNF production by skininfiltrated macrophages $\left(\mathrm{CD} 11 \mathrm{~b}^{+} \mathrm{F} 4 / 80^{+} \mathrm{CD}^{+} 4^{+}\right)$was decreased by $\sim 50 \%$ in $Z c 3 h 12 c^{-1-}$ mice, while TNF production in other myeloid cells remained unchanged (Fig. $4 \mathrm{~g}$, h). Consistent with the germline knockout mice, we observed a reduction of TNF secretion by skin-infiltrating $Z c 3 h 12 c^{\mathrm{M} \Phi \Delta}$ macrophages (Fig. 4i). Here we show that MCPIP3-deficient skin-resident macrophages produce less TNF in psoriatic lesions.

We then investigated the physiological significance of $\mathrm{pDC}$ derived IL-6 in the IMQ model. First, we examined pDC percentages from IMQ-induced psoriatic lesions at day 1 , and found they were comparable between $Z c 3 h 12 c^{+I+} / Z c 3 h 12 c^{-I-}$ mice (Supplementary Fig. 4a, b). Consistently with previously published reports, pDC recruitment to skin peaked within $24 \mathrm{~h}$ after IMQ treatment (Supplementary Fig. 4c) ${ }^{49}$. pDCs and noncanonical DCs (nc-DCs)/transitional DCs (tDCs)/Axl ${ }^{+}$ Siglec- $6^{+}$DCs (ASDCs) share similar surface markers, and these nc-DCs, tDCs, or ASDCs may have been confused for pDCs in previous psoriasis studies ${ }^{50,51}$. Using CX3CR1, a surface marker that differentiates murine $\mathrm{pDCs}$ and $\mathrm{t}-\mathrm{DCs}$, we confirmed that the $\mathrm{CD}_{11 \mathrm{C}^{\text {int }}} \mathrm{B}^{2} 20^{+} \mathrm{Bst}^{+}{ }^{+}$SiglecH ${ }^{+}$cells we identified in IMQinduced lesions at day 1 were CX3CR1 negative (Supplementary Fig. $4 d)^{52}$. Here, we confirm that bona fide pDCs are present early in IMQ-induced psoriatic lesions.

Similar to our pDC experiments in vitro, we found that $Z c 3 h 12 c^{-1-}$ psoriatic skin released more IL-6 than that of $Z c 3 h 12 c^{+1+}$ mice (Fig. 4f). To determine the effect of enhanced IL-6 by pDCs in vivo, we induced psoriatic lesions with IMQ on $Z c 3 h 12 c^{\mathrm{fl} / \mathrm{fl}} / Z c 3 h 12 c^{\mathrm{DC} \Delta}$ mice. Since some macrophages express $\mathrm{CD} 11 \mathrm{c}$, we gated on CD11c negative skin-infiltrating macrophages, and still found a $\sim 25 \%$ reduction in TNF production
(Fig. 4j). Here we show that MCPIP3-deficiency in dendritic cells could extrinsically suppress macrophage TNF.

To imitate the excessive IL- 6 production by pDCs in MCPIPdeficient inflamed skin, we injected WT mice skin with recombinant IL-6 at the beginning of IMQ-induced psoriasis model. We found that intradermal rIL-6 injections above $200 \mathrm{ng}$ significantly reduced the mPASI score of IMQ-treated WT mice (Fig. 4k). High doses of rIL-6 suppressed TNF secretion and Il17a transcripts in MCPIP3-deficient inflamed skin (Fig. 4l, m). Narrowband ultraviolet B (NB-UVB), a standard phototherapy to treat psoriasis, is known to induce copious amount of IL-6 in skin $^{53}$. The amount of IL-6 induced by inflamed skin after UV irradiation is similar to that of IMQ model at day 1 (Fig. $4 \mathrm{n}$ ). We found that $Z c 3 h 12 c$ transcripts in WT mice skin were downregulated after UV irradiation (Fig. 4o). Similarly, rIL-6 injections could lower $Z c 3 h 12 c$ transcripts in IMQ-treated skin (Fig. 4p). Here, we show that intradermal rIL6 injections protect mice against IMQ-induced psoriatic lesions.

MCPIP3-deficiency protects mice from psoriatic skin inflammation. Consistent with the perturbed TNF/IL-12/IL-6 secretion, we found that $Z c 3 h 12 c^{-/-}$mice had $\sim 2$-fold lower murine Psoriasis Severity Index (mPASI) score than $Z c 3 h 12 c^{+/+}$mice at days 3-4, the peak of IMQ-induced psoriatic lesions (Fig. 5a). We observed that the skin of $Z c 3 h 12 c^{-/-}$mice possessed significantly fewer white-scaly desquamations, which is the hallmark of psoriatic lesions (Fig. 5a). After isolating immune cells from the skin lesions, we observed that there were $~ 50 \%$ fewer infiltrated $\mathrm{CD}_{4} 5^{+}$leukocytes, CD11b ${ }^{+} \mathrm{CD}_{11 \mathrm{c}^{-}}$myeloid cells, and $\mathrm{Ly}_{6 \mathrm{G}}{ }^{+}$ Ly6C $^{-}$neutrophils in $Z c 3 h 12 c^{-1-}$ mice, when compared to $Z c 3 h 12 c^{+/+}$littermate controls (Fig. 5b-d). After initial activation by myeloid cells, subsequent IL-17A production by $\mathrm{T}$ cells further accelerates psoriasis pathogenesis ${ }^{27}$. We measured IL-17A production by skin-infiltrating $\mathrm{CD}^{+} 5^{+}$leukocytes and found $~ 50 \%$ decreased IL-17A production in $Z c 3 h 12 c^{-1-}$ mice compared to $Z c 3 h 12 c^{+/+}$mice (Fig. 5e). We also examined $\gamma \delta \mathrm{T}$ cells, the predominant producers of IL-17A during psoriasis. IL-17A production by $\gamma \delta \mathrm{T}$ cells was decreased by $\sim 1$-fold in $Z c 3 h 12 c^{-1-}$ mice compared to $Z c 3 h 12 c^{+l+}$ mice (Fig. 5f). The percentage of dermal $\gamma \delta \mathrm{T}$ cells was also decreased by $\sim 1$-fold in $Z c 3 h 12 c^{-/-}$ mice compared to $Z c 3 h 12 c^{+1+}$ mice (Fig. $5 \mathrm{~g}$ ). Here we find that MCPIP3 promotes myeloid recruitment and $\mathrm{T}$-cell activation in IMQ-induced psoriatic lesions.

However, we found no difference in mPASI clinical score, IL$17 \mathrm{~A}$ production by $\gamma \delta \mathrm{T}$ cells or infiltrated immune cell numbers

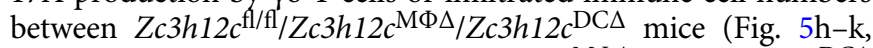
Supplementary Fig. $5 \mathrm{a}-\mathrm{e})$. Since $Z c 3 h 12 c^{\mathrm{M} \Phi \Delta}$ and $Z c 3 h 12 c^{\mathrm{DC} \Delta}$ mice could not exactly phenocopy $Z c 3 h 12 c^{-l-}$ mice in the IMQ model, there is a possibility that unanticipated expression in cells other than pDCs or macrophages may contribute to this phenotype. $\mathrm{CD}_{11 \mathrm{c}^{+}} \mathrm{MHCII}^{+} \mathrm{CD} 1 \mathrm{~b}^{+}$dendritic cells (an heterogenous population consisting of $\mathrm{cDC} 2$ and MoDC) play an important role in psoriasis pathogenesis ${ }^{54}$. We confirm that few MoDCs were present at inflamed skin in the early days of IMQ model, and macrophages were $~ 75 \%$ monocyte-derived (Supplementary Fig. 31).

Second, to exclude the possibility that MCPIP3 regulates IL-17A response in a T-cell-intrinsic manner, we used an recombinant IL23-induced psoriasis model, which sidesteps initial activation by myeloid cell and activates T cells directly via IL- $23^{55}$. We found the genetic ablation of $Z c 3 h 12 c$ did not affect the inflamed ear skin thickness or other immune cell functions, including TNF and IL17A secretion (Fig. 5l-n, Supplementary Fig. 5g-j). Zc3h12c mRNA was little expressed in various T-cell subsets (Fig. 1f, Supplementary Fig. 5k). Using a Th17-driven experimental autoimmune 
a

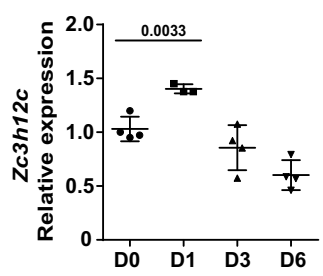

d

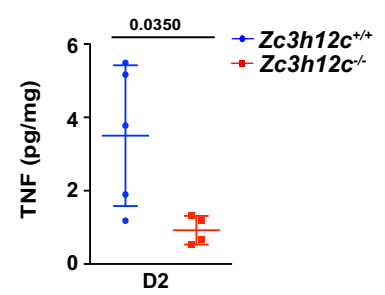

g

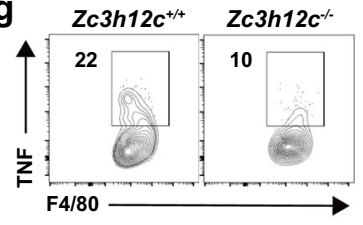

b

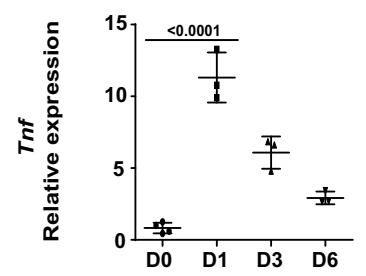

e

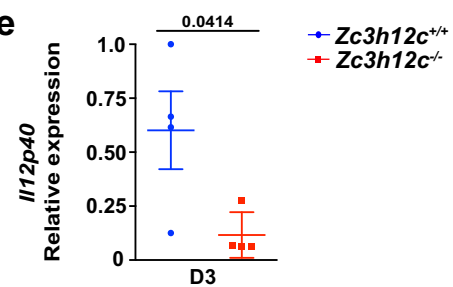

C

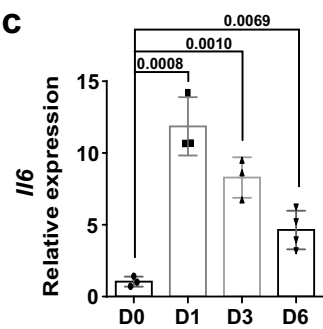

$\mathbf{f}$

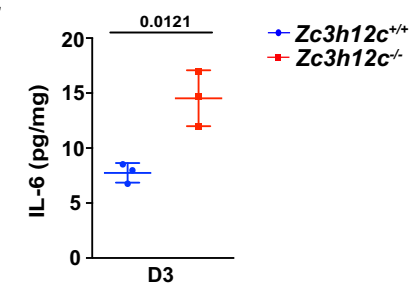

i Zc3h12c $c^{f f f t} \quad Z c 3 h 12 c^{M \Phi \Delta}$
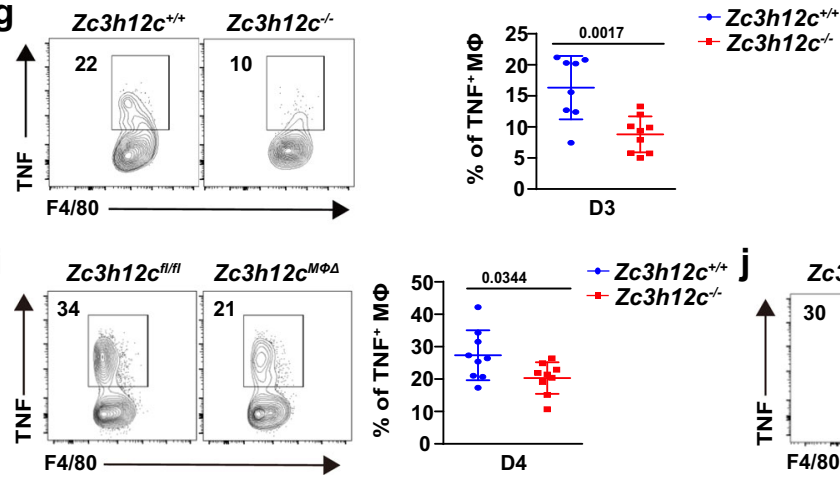

h
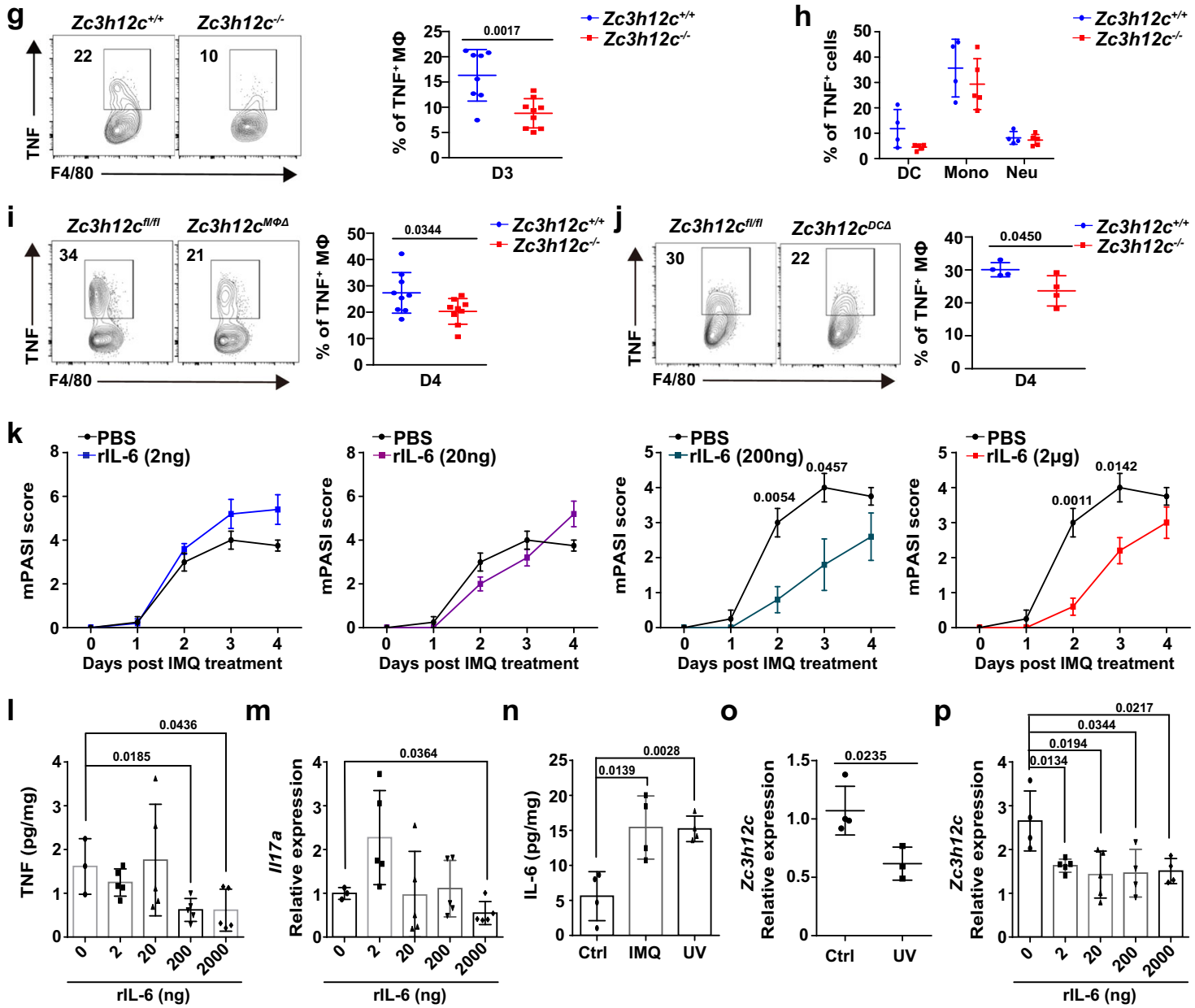

m

n

$\mathbf{0}$
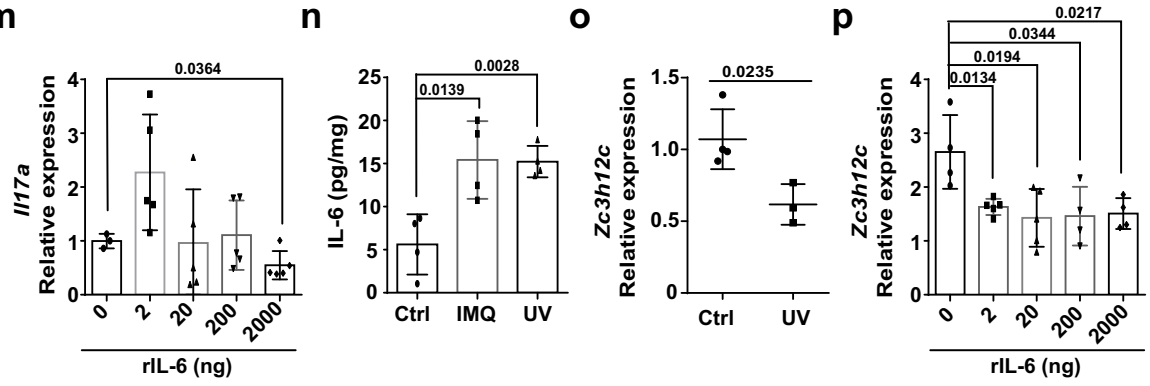

encephalomyelitis (EAE) model, we found that the EAE clinical score and Th17 responses in $Z c 3 h 12 c^{+/+}$and $Z c 3 h 12 c^{-1-}$ mice were similar (Fig. 5o, Supplementary Fig. 5l). Thus, MCPIP3 regulates IL-17A production in a T-cell-extrinsic manner.

Lastly, to exclude the possibility that any expression of $Z c 3 h 12 c$ in nonhematopoietic cells, such as keratinocytes, langerin cells, and tissue-resident macrophages, may play a role in psoriasis, we generated reverse chimeras from $Z c 3 h 12 c^{-1-}$ mice (Supplementary Fig. $5 \mathrm{~m}, \mathrm{n})$. We found that irradiated $Z c 3 h 12 c^{+7+}$ mice reconstituted with $Z c 3 h 12 c^{-1-}$ bone marrow $\left(Z c 3 h 12 c^{-1-}\right.$ to CD45.1) had similar mPASI score as $Z c 3 h 12 c^{-1-}$ mice, whereas irradiated $Z c 3 h 12 c^{-1-}$ mice reconstituted with $Z c 3 h 12 c^{+/+}$bone marrow (CD45.1 to $Z c 3 h 12 c^{-/-}$) had similar mPASI score as $Z c 3 h 12 c^{+l+}$ mice (Fig. 5p). Straight chimeras $\left(Z c 3 h 12 c^{-l-}\right.$ to CD45.1) phenocopied $Z c 3 h 12 c^{-1-}$ mice in terms of infiltrated leukocytes; whereas reverse chimeras (CD45.1 to $Z c 3 h 12 c^{-/-}$) did not (Supplementary Fig. 5o). Most importantly, skin-infiltrated macrophages from straight chimeras $\left(Z c 3 h 12 c^{-1-}\right.$ to CD45.1) exhibited $\sim 50 \%$ decrease in TNF production, when compared with reverse chimeras (CD45.1 to $Z c 3 h 12 c^{-1-}$ ) (Fig. 5q). Thus, MCPIP3-deficiency in cells of hematopoietic origin is necessary and sufficient to protect mice from psoriatic lesions. Collectively, 
Fig. 4 Enhanced intradermal IL-6 alleviates psoriasiform skin inflammation. a-c Zc3h12c, Tnf, and II6 expression in imiquimod (IMQ)-treated skin of WT mice at indicated days was measured by qPCR (a: $n=4$ mice for day 0 and $3, n=3$ mice for day 1 and $6 ; \mathbf{b}: n=4$ mice for day $0, n=3$ mice for day 1,3 , and 6; c: $n=3$ mice for day 0,1 , and 3, $n=4$ mice for day 6). d TNF secretion by IMQ-treated skin of $Z c 3 h 12 c^{+/+}$(blue)/Zc3h12c-/- (red)mice at day 2 was measured by ELISA after normalizing with weight ( $n=5$ mice per group). e $/ 112 \mathrm{p} 40$ expression of IMQ-treated skin of $Z \mathrm{c} 3 \mathrm{~h} 12 \mathrm{c}^{+/ /} / \mathrm{Zc} 3 \mathrm{~h} 12 \mathrm{c}^{-/} /-$mice at day 3 was measured by qPCR ( $n=4$ mice per group). $\mathbf{f} I \mathrm{~L}-6$ secretion by IMQ-treated skin of $Z \mathrm{c} 3 \mathrm{~h} 12 \mathrm{c}^{+/}+/ \mathrm{Zc} 3 \mathrm{~h} 12 \mathrm{c}^{-/-}$mice at day 3 was measured by ELISA after normalizing with weight ( $n=3$ mice per group). $\mathbf{g}$, h TNF production by infiltrated myeloid cells from $Z c 3 h 12 c^{+/+} / Z c 3 h 12 c^{--/}-I M Q-t r e a t e d$ skin on day 3 was measured by intracellular staining after R848 activation (g: $n=8$ mice for Zc3h12c $c^{+/+} ; n=9$ mice for $Z c 3 h 12 c^{-/-} ; \mathbf{h}: n=5$ mice per group). i TNF production by infiltrated macrophages (CD45+ $\left.\mathrm{CD}_{11 b^{+}} \mathrm{F} 4 / 80^{+} \mathrm{CD} 64^{+}\right)$from Zc3h12fl/fl $/ Z c 3 h 12 c^{\mathrm{M} \Phi \Delta}$ IMQ-treated skin on day 4 was measured by intracellular staining after R848 activation ( $n=9$ mice per group). $\mathbf{j}$ TNF production by infiltrated macrophages (CD45+ CD11 ${ }^{-}$CD11b ${ }^{+}$F4/

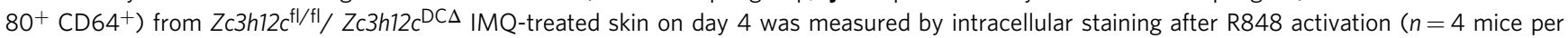
group). $\mathbf{k}$ Psoriasis severity of IMQ-treated WT mice with rlL-6 injections were scored by mPASI daily ( $n=5$ mice per group). I TNF secretion by IMQ + rIL-6-treated skin at day 3 was measured by ELISA after normalizing with weight ( $n=5$ mice per group). $\mathbf{m}$ II17a expression in IMQ + rIL-6 treated skin of WT mice at indicated days was measured by qPCR ( $n=5$ mice per group). $\mathbf{n}$ IL- 6 secretion by treated skin was measured by ELISA after normalizing with weight ( $n=4$ mice per group). o Zc3h12c expression in UV-treated skin at $24 \mathrm{~h}$ was measured by qPCR ( $n=5$ mice per group). $\mathbf{p}$ Zc3h12c expression in IMQ + rlL-6-treated skin at day 4 was measured by qPCR ( $n=5$ mice per group). Data are representative of at least three independent experiments (mean \pm S.D.; two-tailed Student's $t$ test). Source data are provided as a Source Data file.

these data suggest that simultaneous TNF repression and IL-6 enhancement, by MCPIP3-deficient macrophages and dendritic cells respectively, could protect mice against IMQ-induced psoriatic lesions.

Exposure to pDC-derived IL-6 suppresses macrophage TNF and IL-12p40. It was previously shown that IL-6 from conventional dendritic cells could inhibit the production of inflammatory cytokines by inhibiting NFkb and inducing soluble p55 receptor for $\mathrm{TNF}^{56}$. Since macrophage TNF was downregulated in $Z c 3 h 12 c^{\mathrm{DC} \Delta}$ psoriatic lesions (Fig. 4j), we hypothesize that pDCs could directly regulate macrophage TNF secretion. After co-culturing WT FL3TLpDCs with WT BMDMs, we found that TNF secretion by R848stimulated macrophages was blocked by $\sim 5$-fold with equal numbers of co-cultured pDCs (Fig. 6a). It was worth noting that TNF was decreased by $\sim 30 \%$ with $1-10 \%$ pDCs, which was within range of pDC percentage amongst immune cells at psoriatic skin on day 1 (Fig. 6a, Supplementary Fig. 4a). To determine if IL-6 could suppress macrophage TNF, we treated WT BMDMs with various concentration of recombinant IL-6 along with R848, and then measured their TNF or IL-12p40 production by intracellular staining. We found that initial exposure to IL-6 could lower macrophage TNF by $\sim 15 \%$, and IL- $12 \mathrm{p} 40$ production by $\sim 30 \%$ (Fig. $6 \mathrm{~b}$ ). Next, we added supernatants of activated WT FL3TL-pDCs (pDC ${ }^{\text {sup }}$ ) to $Z c 3 h 12 c^{+l+} / Z c 3 h 12 c^{-l-}$ BMDMs, and then measured macrophage TNF production at $4 \mathrm{~h}$ by intracellular staining. As a comparison, we added recombinant IL-6. Supernatants from FL3TL-pDCs lowered TNF production of $Z c 3 h 12 c^{+/+}$and $Z c 3 h 12 c^{-1-}$ macrophages by $\sim 15 \%$, similar to IL-6 (Fig. 6c). This suppressive effect was absent at late-phase of macrophage activation (Fig. 6d). We repeated the same experiments with FACS-sorted BM pDCs, and found that the difference between $Z c 3 h 12 c^{+l+} / Z c 3 h 12 c^{-I-} \mathrm{TNF}^{+}$macrophages now reached $\sim 50 \%$ (Fig. 6 e). We were finally able to recreate this substantial TNF reduction in vitro, similar to what was observed in vivo at psoriatic lesions (Fig. 4g). Similar to WT pDCs, $Z c 3 h 12 c^{-1-} \mathrm{pDC}$ supernatant suppressed macrophage TNF, and this suppressive effect was abolished with addition of neutralizing anti-IL-6 monoclonal IgG antibodies (Fig. 6f). pDC supernatants suppressed macrophage IL-12p40 in a similar manner (Fig. 6g, h). Here we show in vitro that early exposure to pDC-derived IL- 6 suppresses macrophage TNF and IL-12p40 production.

pDC secret copious amount of IFN-I, which was known to regulate TNF production in various cell types ${ }^{3}$. IFN- $\alpha$ suppression of macrophage TNF was reported under $\mathrm{FcR} \varepsilon$ ligation and Pam3CSK stimulation, but the effect under R848 was not explored $^{57}$. First, we observed that supernatant from CpG A- activated FL3TL-pDCs could not suppress macrophage TNF at all (Supplementary Fig. 41). Second, we could not find any IFN-a suppression of TNF in R848-activated macrophages with ELISA or intracellular staining, even at high concentrations $(1 \mathrm{mg} / \mathrm{ml})$ (Supplementary Fig. 4m, n). Lastly, we induced psoriasis with IMQ on double transgenic Ifnar $1^{-1-}: Z c 3 h 12 c^{-l-}$ mice. We found that Ifnar $1^{-1-}: Z c 3 h 12 c^{-1-}$ mice maintained the same phenotype as Ifnar $1^{-+/+}: Z c 3 h 12 c^{-/-}$mice, in terms of ear thickness, infiltrated immune cells and IL-17A production (Supplementary Fig. 4o, p). Our data show that suppression of R848-activated macrophage TNF by pDCs is independent of IFNI signaling.

MCPIP3 directly degrades IL-6 and Regnase-1 mRNAs. Since MCPIP3 contains putative zinc finger CCCH RNA-binding and NYN RNase domains, we sought to identify specific degradative targets of MCPIP3 in pDCs and macrophages. Because we already found that IL-6 protein and mRNA was upregulated in MCPIP3deficient pDCs, we hypothesized that MCPIP3 might directly degrade IL-6 mRNAs. For this purpose, we mutated possible enzymatically active sites (D271N, D271A, D356A, S372A, $\mathrm{D} 374 \mathrm{~A}$, and D378A) at the predicted RNA-binding groove of NYN nuclease domain of MCPIP3. As a positive control, we utilized Regnase-1, a known IL-6 mRNA degrader, and its functionally inactive D141N or C306R mutants (Fig. 7a).

First, we measured secreted IL- 6 in the supernatant after cotransfecting 293T cells with IL-6 and MCPIP3 overexpression plasmids. MCPIP3 could lower IL-6 concentrations in the supernatant, albeit not as efficient as Regnase-1 (Fig. 7b). Overexpression by MCPIP3 D271N mutant was able to rescue IL-6 secretion, whereas S372A mutant could not (Fig. 7b). In a negative control using Il6 CDS without $3^{\prime} \mathrm{UTR}$, neither MCPIP3 nor its mutants could not have any effects on IL- 6 concentrations (Fig. 7c). Second, we cloned the IL-6 $3^{\prime} \mathrm{UTR}$ region into a luciferase reporter system, along with a MCPIP3 overexpression plasmid. Similar to Regnase-1, MCPIP3 was able to abolish reporter signals from IL-6 3'UTRs constructs (Fig. 7d). Consistently, MCPIP3 D271N mutant failed to abolish IL-6 reporter signals (Fig. 7d). To address the concern that MCPIP3 may be an unspecific RNase, we utilized $3^{\prime}$ UTR of several negative control mRNAs such as $\beta$-actin, Hprt, or Ccr7. We found that their luciferase activity was unaffected by MCPIP3 overexpression (Fig. 7e). Lastly, total IL-6 mRNA was reduced in presence of MCPIP3 (Fig. 7f). These results suggest that MCPIP3 uses its NYN nuclease domain to degrade IL-6 mRNA by binding to its $3^{\prime}$ UTR. 
a
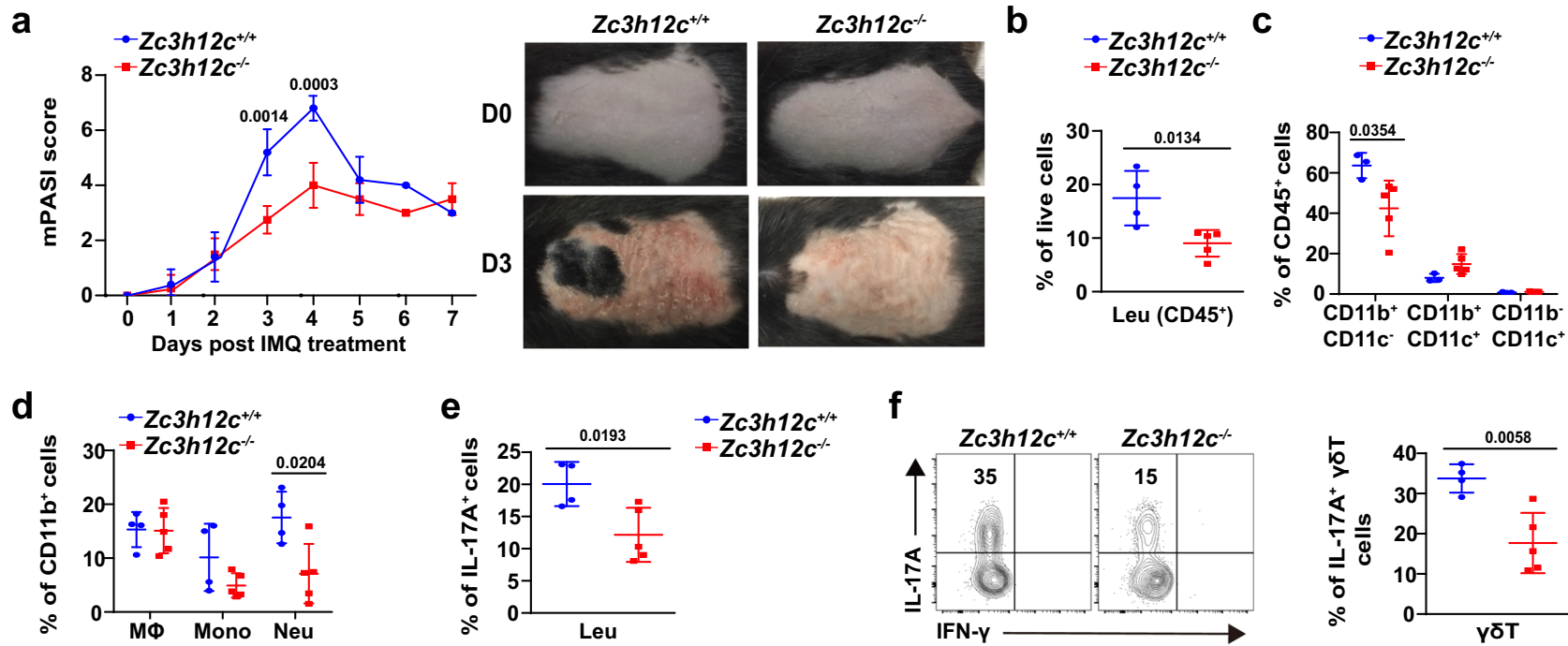

f
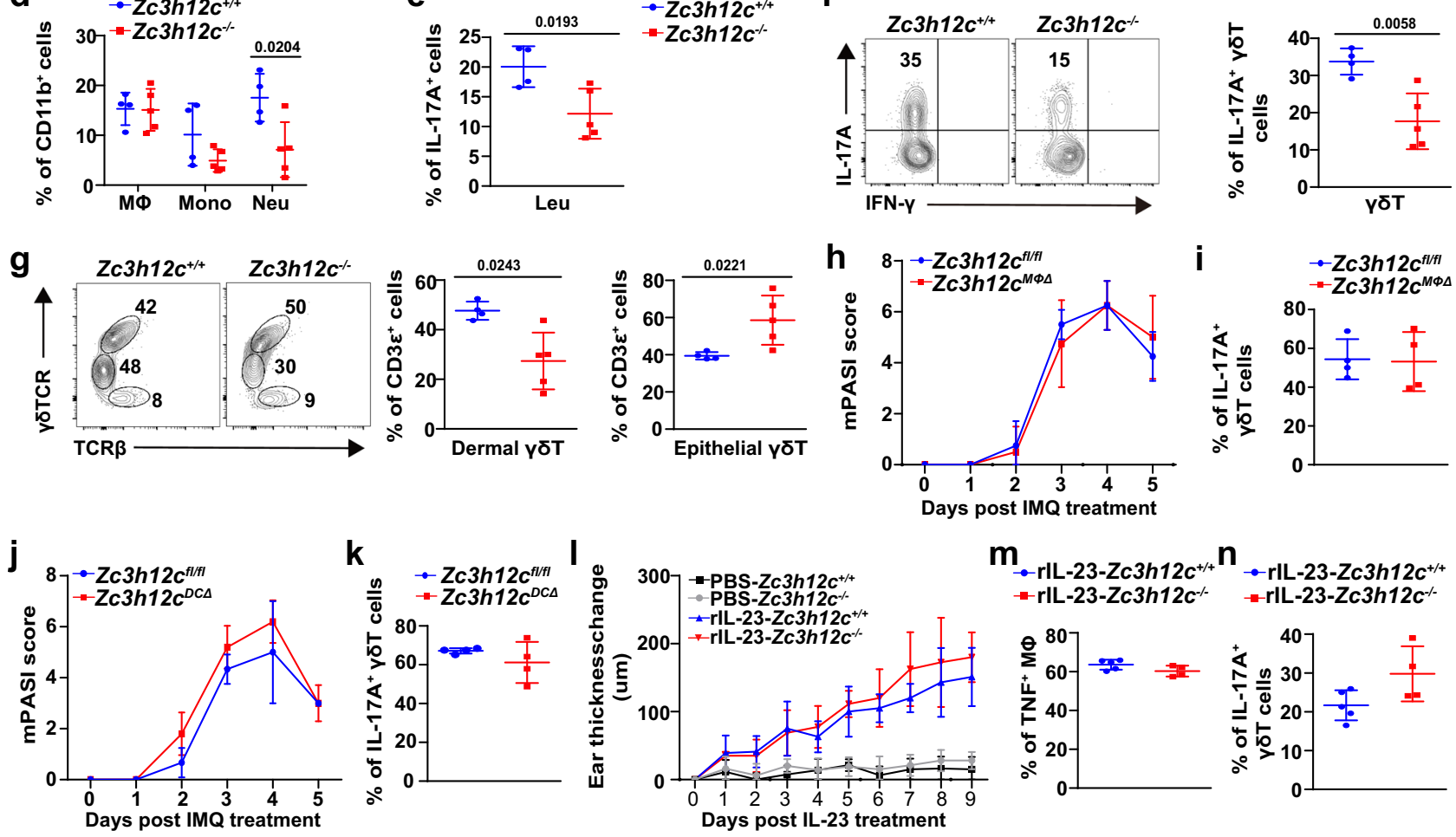

O

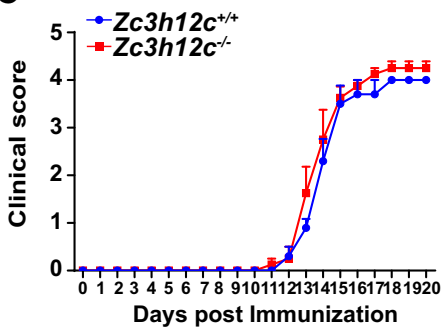

p

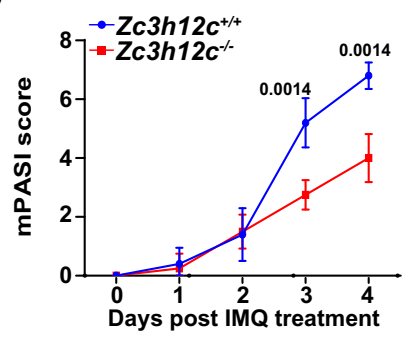

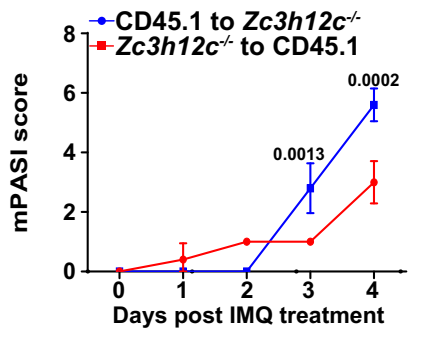

$\mathbf{q}$

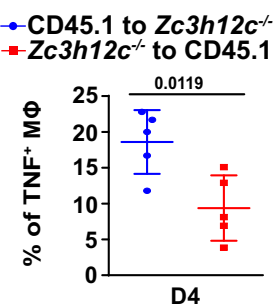

Besides IL-6, we found that a close family member, $Z c 3 h 12 a$ (the gene encoding Regnase-1/MCPIP1/ZC3H12A), was upregulated in $Z c 3 h 12 c^{-1-}$ BMDMs (Fig. $7 g$ ). By comparison, the mRNA expression of MCPIP2 (Zc3h12b) and MCPIP4 (Zc3h12d) were not affected in MCPIP3-deficient macrophages (Supplementary Fig. 6b, c). To exclude the possibility that MCPIP1's upregulation is due to genetic compensation from point mutations in $Z c 3 h 12 c^{-1-}$ mice, we examined $Z c 3 h 12 a$ mRNA in Per. MФs of $Z c 3 h 12 c^{\mathrm{M} \Phi \Delta}$ mice, in which an entire exon was deleted. $Z c 3 h 12 a$ mRNA was also upregulated in $Z c 3 h 12 c^{\mathrm{M} \Phi \Delta}$ macrophages (Fig. $7 \mathrm{~h}$ ). In pDCs, we detected an increase of Zc3h12a mRNA, albeit only after R848 or CpG B activation (Fig. 7i). Here we demonstrate that Regnase-1 mRNA is upregulated in MCPIP3-deficient macrophages and pDCs.
To determine whether MCPIP3 could bind and degrade Regnase- 1 mRNA, we cloned the $Z c 3 h 12 a 3^{\prime}$ UTR region into a luciferase reporter system, along with a MCPIP3 overexpression plasmid. We found that $293 \mathrm{~T}$ cells transfected with $Z c 3 h 12 a 3^{\prime}$ UTRs showed $\sim 4$-fold less luciferase activity in the presence of MCPIP3 (Fig. 7j). The MCPIP3 D271N mutant was able to restore $Z c 3 h 12 a$ reporter signals, suggesting this could be a key site for nuclease activity (Fig. 7j). Second, we constructed tagged Regnase-1 (MYC) and MCPIP3 (FLAG), and examined Regnase1 protein level in the presence of MCPIP3 protein. When MCPIP3 was co-transfected with $Z c 3 h 12 a$ CDS with its $3^{\prime} \mathrm{UTR}$, less amount of Regnase- 1 protein was found (Fig. 7k). In contrast, presence of MCPIP3 did not affect Regnase-1 when cotransfecting with $Z c 3 h 12 a$ CDS only without its $3^{\prime} \mathrm{UTR}$ (Fig. 7k). 
Fig. 5 MCPIP3-deficiency protects mice from psoriatic skin inflammation. a Psoriasis severity of imiquimod (IMQ)-treated skin from Zc3h12c $+/+(b l u e) /$ $Z c 3 h 12 c^{-/}$(red)mice was measured daily by mPASI ( $n=4$ mice per group). Representative pictures of the treated back-skin were shown at right. b-d Percentages of infiltrated immune cells from IMQ-treated $Z \mathrm{c} 3 \mathrm{~h} 12 \mathrm{c}^{+/} / \mathrm{Z} / \mathrm{Zc} 3 \mathrm{~h} 12 \mathrm{c}^{-/}$- back-skin on day 3 were analyzed by flow cytometry $(n=4$ mice for

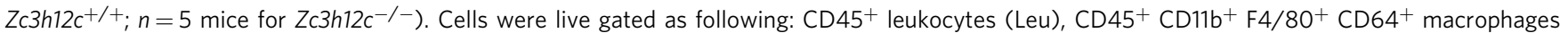
$(\mathrm{M} \Phi), \mathrm{CD} 45^{+} \mathrm{CD} 11 \mathrm{~b}^{+} \mathrm{Ly} 6 \mathrm{G}+$ neutrophils (Neu), CD45+ CD11b+ $\mathrm{Ly}^{+} \mathrm{C}^{+}$monocytes (Mono). e, f $\mathrm{IL}-17 \mathrm{~A}$ production by $\mathrm{CD} 45^{+}$leukocytes $(\mathbf{e})$ and $\mathrm{CD} 3 \varepsilon^{+}$ $\gamma \delta \mathrm{STCR}^{+} \mathrm{T}$ cells $(\mathbf{f})$ at $Z \mathrm{c} 3 \mathrm{~h} 12 \mathrm{c}^{+/+} / \mathrm{Zc} 3 \mathrm{~h} 12 \mathrm{c}^{-/-} \mathrm{IMQ}$-treated skin on day 3 was measured by intracellular staining $\left(n=4\right.$ mice for $Z \mathrm{c} 3 \mathrm{~h} 12 \mathrm{c}^{+/+} ; n=5 \mathrm{mice}$ for $\left.Z c 3 h 12 c^{-/-}\right) . \mathbf{g} \gamma \delta$ T-cell subset composition at IMQ-treated $Z c 3 h 12 c^{+/+} / Z c 3 h 12 c^{-/-}$back-skin on day 3 was analyzed by flow cytometry $(n=4$ mice for $Z c 3 h 12 c^{+/+} ; n=5$ mice for $\left.Z c 3 h 12 c^{-/-}\right)$. Dermal $\left(\gamma \delta T C R^{+} T C R \beta^{-}\right)$and epithelial $\left(\gamma \delta T C R^{+} T C R \beta^{+}\right) \gamma \delta$ T-cell subsets were gated from live ${ }^{+}$CD45 $5^{+}$

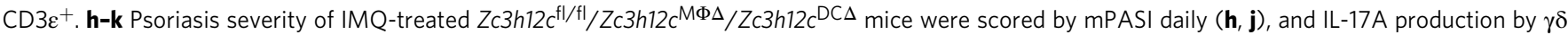
T cells $(\mathbf{i}, \mathbf{k})$ at day 4 was measured $\left(n=4\right.$ mice per group). I Skin thickness of $\mathrm{rlL}-23$-treated ears of $Z \mathrm{c} 3 \mathrm{~h} 12 \mathrm{c}^{+/ /}+/ \mathrm{Zc} 3 \mathrm{~h} 12 \mathrm{c}^{-/-}$mice was measured daily $\left(n=5\right.$ mice for $Z c 3 h 12 c^{+/+} ; n=4$ mice for $\left.Z c 3 h 12 c^{-/-}\right)$. $\mathbf{m}$ TNF production by infiltrated macrophages rlL-23-treated ears of $Z c 3 h 12 c^{+/+} / Z c 3 h 12 c^{-/-}$ mice at day $9\left(n=5\right.$ mice for $Z c 3 h 12 c^{+/+} ; n=4$ mice for $\left.Z c 3 h 12 c^{-/-}\right)$. $\mathbf{n}$ IL-17A production by $\gamma \delta \mathrm{T}$ cells at rlL-23-treated ear on day 9 was measured by intracellular staining ( $n=5$ mice for $Z c 3 h 12 c^{+/+} ; n=4$ mice for $Z c 3 h 12 c^{-/-}$). o EAE score of $Z c 3 h 12 c^{+/+} / Z c 3 h 12 c^{-/-}$mice $(n=5$ mice per group). p Psoriasis severity of the chimeras were scored by mPASI daily ( $n=5$ mice per group). $\mathbf{q}$ TNF production by infiltrated macrophages from the chimeras at day 4 was measured by intracellular staining after R848 activation ( $n=5$ mice per group). Data are representative of five (a-g), or two (h-q) independent experiments (mean \pm S.D.; two-tailed Student's $t$ test). Source data are provided as a Source Data file.
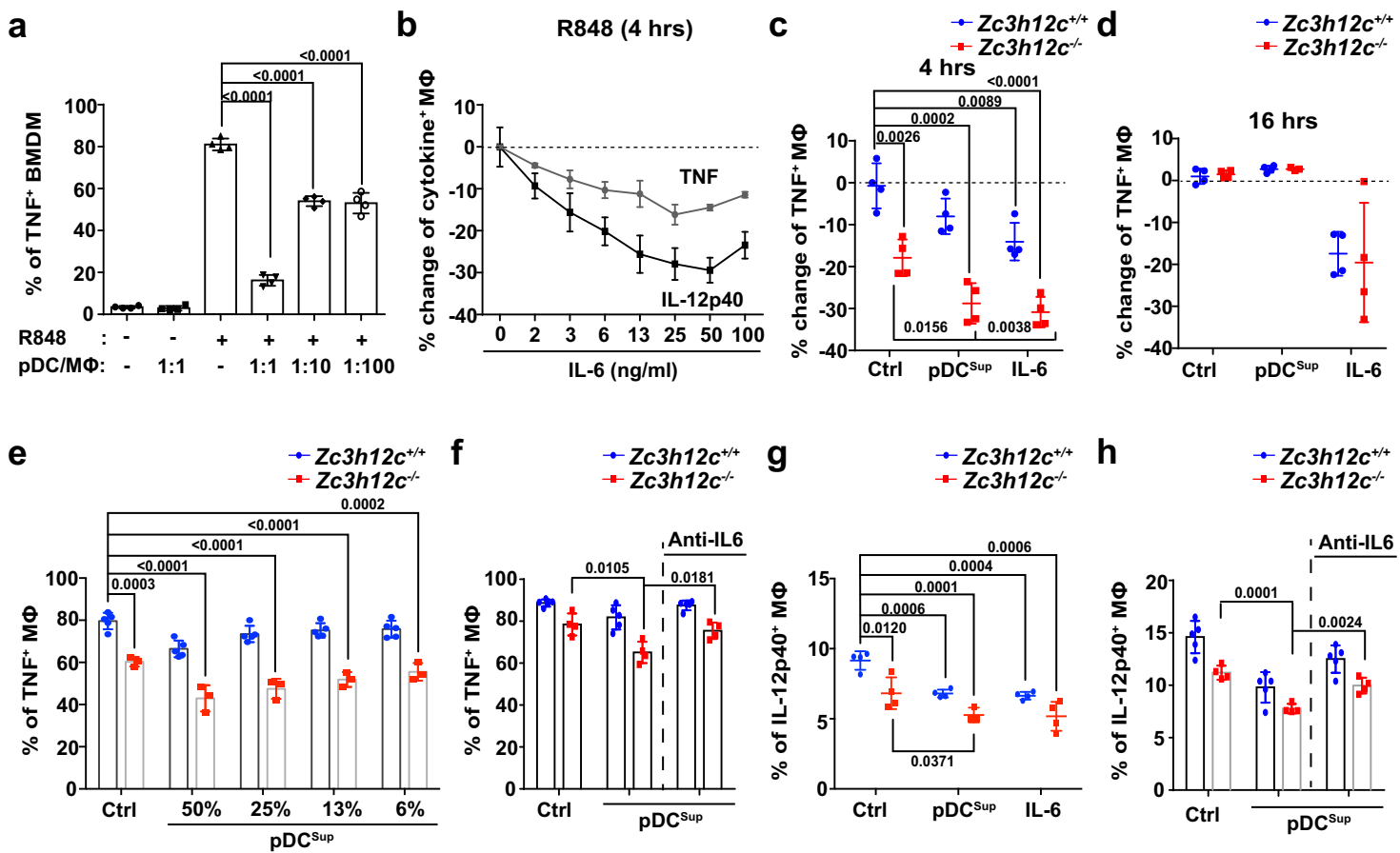

Fig. 6 Exposure to pDC-derived IL-6 suppresses macrophage TNF and IL-12p40. a Sorted WT bone marrow-derived macrophages (BMDMs) and FL3TLpDCs were placed into the same wells for $16 \mathrm{~h}$ before addition of R848. TNF production in macrophages was measured by intracellular staining in macrophages at $4 \mathrm{~h}$ ( $n=4$ mice per group). b WT BMDMs were treated with recombinant IL-6 at various concentrations, and macrophage TNF or IL-12p40 production was measured by intracellular staining at $4 \mathrm{~h}$. Percentage changes was normalized against WT samples without IL-6 ( $n=3$ mice per group). c, d Sorted WT FL3TLpDCs were treated with R848 for $24 \mathrm{~h}$, and their supernatants (pDCsup) or rlL-6 (50 ng/ml) were added to Zc3h12c $\mathrm{c}^{+/+}$(blue)/Zc3h12c ${ }^{-/-}$(red)BMDMs along with R848. TNF production in macrophages at $4 \mathrm{~h}(\mathbf{c})$ or $16 \mathrm{~h}(\mathbf{d})$ was measured by intracellular staining. Percentage changes was normalized against $Z c 3 \mathrm{~h} 12 \mathrm{c}^{+} /+$

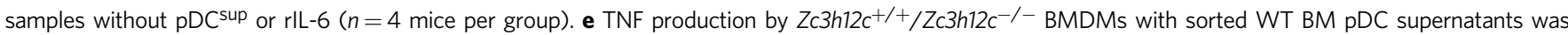
measured by intracellular staining after R848-treatment at $4 \mathrm{~h}$ ( $n=5$ mice per group). f TNF production by $Z \mathrm{c} 3 \mathrm{~h} 12 \mathrm{c}^{+/} /+/ \mathrm{Zc} 3 \mathrm{~h} 12 \mathrm{c}^{-/-}$BMDMs with titrated concentrations of R848-treated sorted Zc3h12c-/- FL3TL-pDC supernatants, with or without IL-6 neutralizing antibodies was measured by intracellular staining

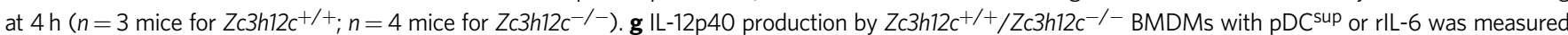
by intracellular staining at $4 \mathrm{~h}\left(n=5\right.$ mice for $Z \mathrm{c} 3 \mathrm{~h} 12 \mathrm{c}^{+/+} ; n=4$ mice for $\left.Z \mathrm{c} 3 \mathrm{~h} 12 \mathrm{c}^{-/-}\right)$. $\mathbf{h} \mathrm{IL}-12 \mathrm{p} 40$ production by $Z \mathrm{c} 3 \mathrm{~h} 12 \mathrm{c}^{+/+} / \mathrm{Zc} 3 \mathrm{~h} 12 \mathrm{c}^{-/-}$BMDMs with titrated concentrations of R848-treated sorted Zc3h12c-/- FL3TL-pDC supernatants, along with or without IL-6 neutralizing antibodies was measured by intracellular staining at $4 \mathrm{~h}(n=4$ mice per group). Data are representative of five (c, $\mathbf{d})$, three $(\mathbf{g})$, or two $(\mathbf{a}, \mathbf{b}, \mathbf{e}, \mathbf{f}, \mathbf{h})$ independent experiments (mean \pm S.D.; two-tailed Student's $t$ test). Source data are provided as a Source Data file.

Our results suggest that MCPIP3 specifically binds to Zc3h12a $3^{\prime}$ UTR, and degrades Regnase-1 mRNA with its nuclease domain.

MCPIP3 regulates cytokine secretion via Regnase- 1 and NFkB pathway. Regnase-1 exerts its TNF suppression by degrading
mRNA of NFkB signaling molecules such as c-Rel and IкB $\zeta$, and possibly TNF itself $8,14,20,58$. Regnase-1-deficient mice exhibited excessive TNF secretion during septic shock, and Regnase-1 could bind to a stem loop at CDE37 region of Tnf $3^{\prime} \mathrm{UTR}$ in luciferase reporter assays ${ }^{14,58}$. We confirmed the direct degradation of TNF by Regnase- 1 by measuring secreted TNF with ELISA, after 
a

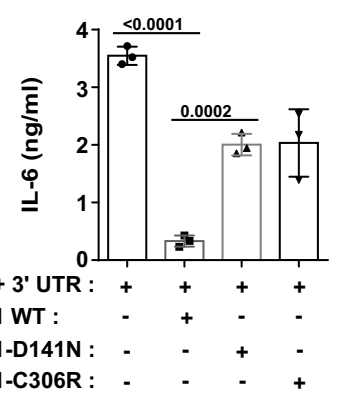

b

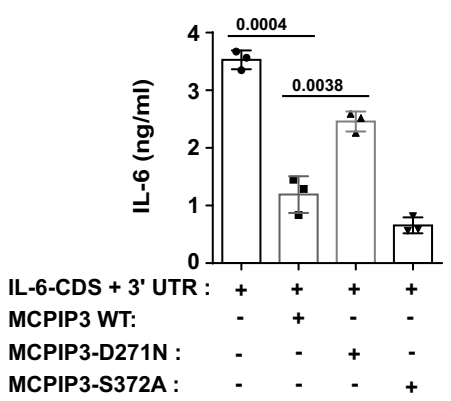

C

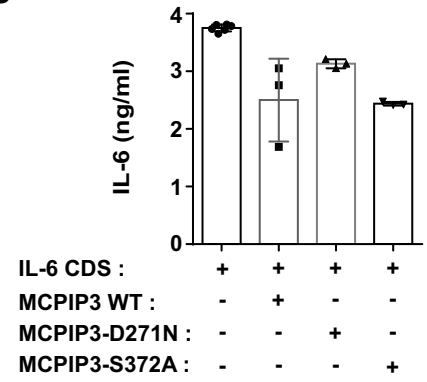

d

II6 3' UTR

e

$\beta$-actin 3' UTR

Hprt 3' UTR

Ccr7 3' UTR
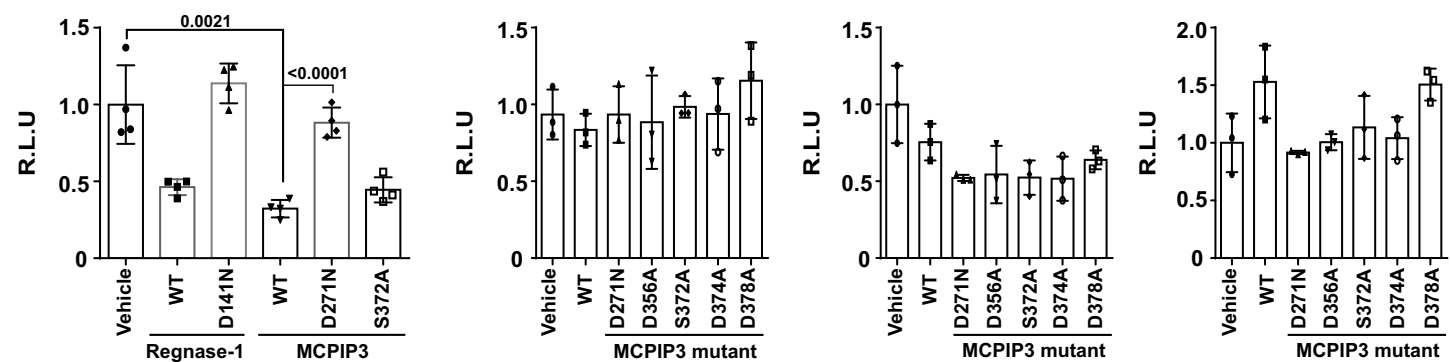

f

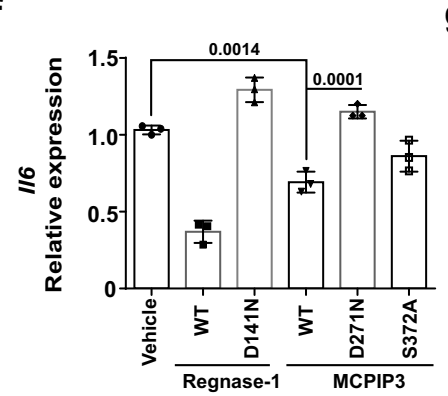

g
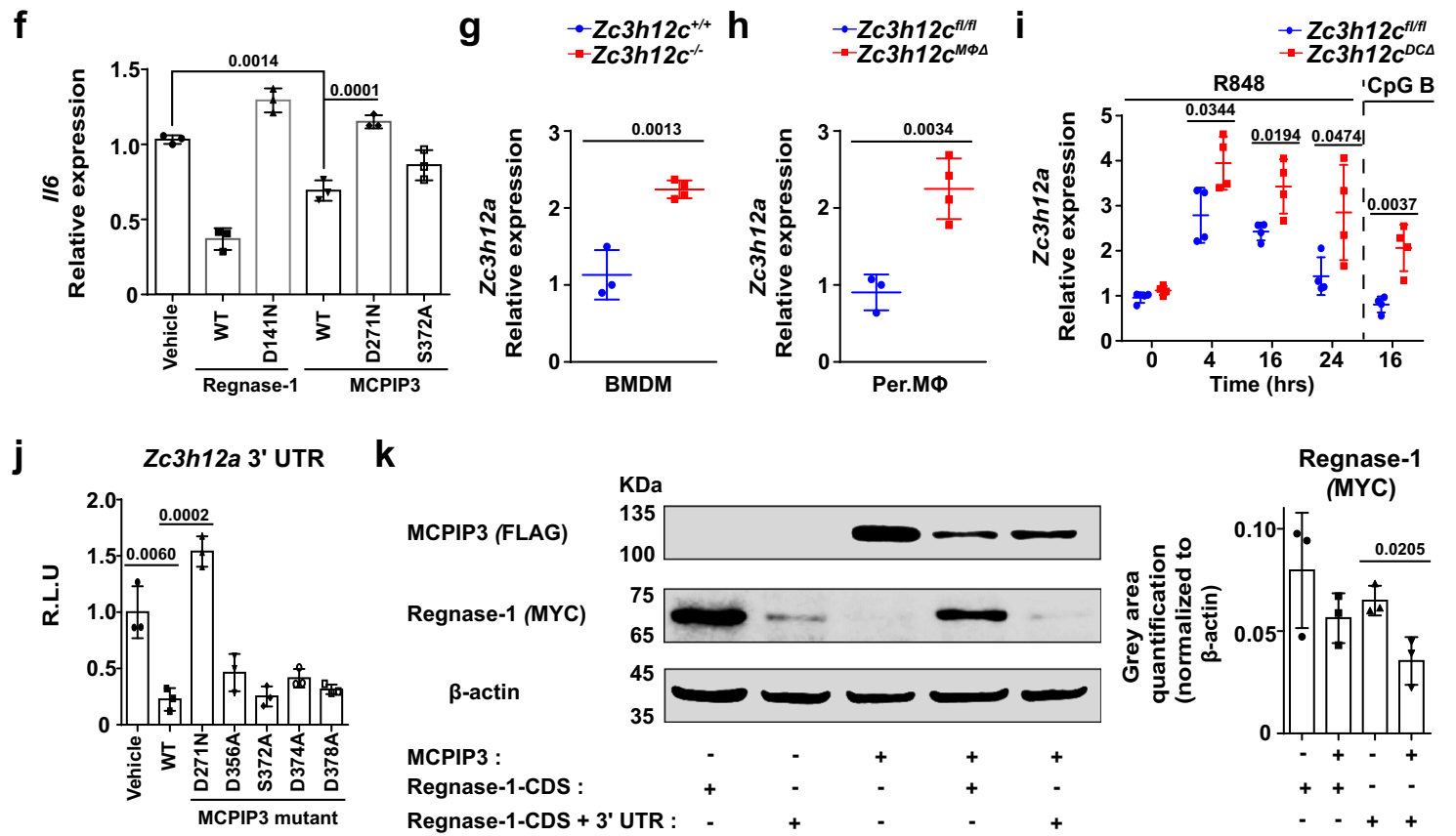

k
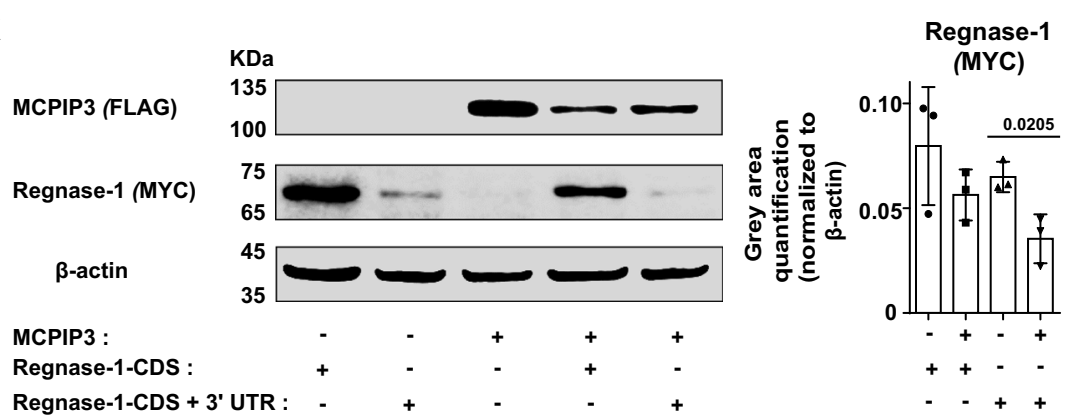

Fig. 7 MCPIP3 directly degrades IL-6 and Regnase-1 mRNAs. a-c 293T cells were co-transfected with Regnase-1 (a) or MCPIP3 (b, c), and IL-6 CDS with $3^{\prime}$ UTR $(\mathbf{a}, \mathbf{b})$ or without 3' UTR (c). After 48 h, IL-6 in supernatants were measured by ELISA ( $n=4$ replicates for IL-6 CDS only; $n=3$ replicates for all other groups). d Degradation of 116 mRNA by MCPIP3 was measured by a luciferase reporter system. 3' UTR of 116 were inserted downstream of the luciferase gene. These reporter plasmids were then transfected into 293T cells, along with empty, MCPIP3 or mutant MCPIP3 overexpression plasmids ( $n$ $=4$ replicates). e 3' UTR of negative control genes ( $\beta$-actin, Hprt, Ccr7) were inserted downstream of the luciferase gene. $\mathbf{f} 293 \mathrm{~T}$ cells were co-transfected with MCPIP3 and IL-6 CDS with 3' UTR. After $48 \mathrm{~h}$, 116 mRNA in transfected cells were measured by qPCR $(n=3$ replicate wells). $\mathbf{g}$ Zc3h12a (the gene encoding Regnase-1) expression in sorted $Z c 3 h 12 c^{+/+} / Z c 3 h 12 c^{-/-}$bone marrow-derived macrophages (BMDMs) was measured by qPCR ( $n=3$ mice for $Z c 3 h 12 c^{+/+} ; n=4$ mice for $\left.Z c 3 h 12 c^{-/-}\right)$. h Zc3h12a expression in purified Zc3h12ctl/fl $/ Z c 3 h 12 c^{M \Phi \Delta}$ peritoneal macrophages (Per. M $\Phi$ ) was measured by qPCR ( $n=3$ mice for $Z c 3 h 12 c^{+/+} ; n=4$ mice for $Z c 3 h 12 c^{-/-}$). i Zc3h12a expression in sorted Zc3h12 fl/fl $/ Z c 3 h 12 c^{D C \Delta}$ bone marrow (BM) pDCs was measured by qPCR ( $n=4$ mice per group). $\mathbf{j}$ Degradation of $Z c 3 h 12 a$ mRNA by MCPIP3 was measured by a luciferase reporter system ( $n=3$ replicates). k 293 T cells were transfected with FLAG-tagged MCPIP3 together with MYC-tagged Regnase-1. After 48 h, whole cell lysates were immunoprecipitated with anti-MYC or FLAG antibodies. Gray area quantification of the Regnase-1-MYC was calculated by ImageJ, after normalizing with $\beta$-actin bands ( $n=3$ replicate bands). The samples were derived from the same experiment and that gels/blots were processed in parallel. Data are representative of at least three independent experiments (mean \pm S.D.; two-tailed Student's $t$ test). Source data are provided as a Source Data file. 

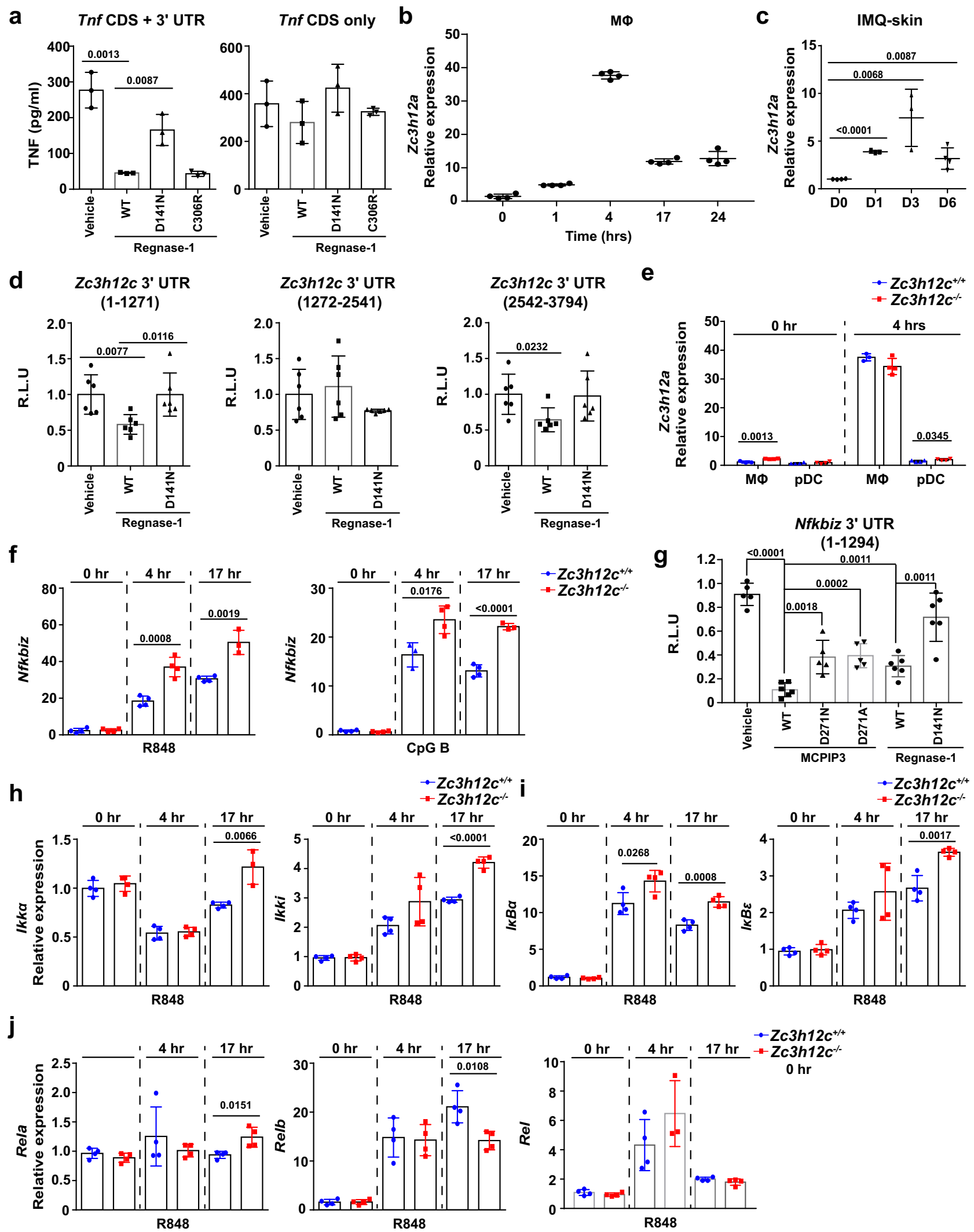

co-transfecting Zc3h12a and Tnf overexpression plasmids (Fig. 8a). Thus, MCPIP3 could promote TNF expression by degrading Regnase-1, a negative TNF regulator.

It remains puzzling how TNF and IL-6 was regulated distinctively by MCPIP3 in macrophages and pDCs. In macrophages, Regnase-1 upregulation coincided with the that of
MCPIP3 after $4 \mathrm{~h}$ (Fig. 8b). In psoriatic skin, Regnase-1 upregulation peaked at day 3, rather than day 1 for MCPIP3 (Fig. 8c). To determine if Regnase-1 could degrade MCPIP3 mRNA, we utilized luciferase reporter plasmids inserted with MCPIP3 3'UTR, along with Regnase-1 plasmids. We found that Regnase-1 was able to decrease luciferase reporter activity by 
Fig. 8 MCPIP3 regulates cytokine secretion via Regnase-1 and NFKB pathway. a 293T cells were co-transfected with Regnase-1, TNF-CDS with 3' UTR or without 3' UTR. After $48 \mathrm{~h}$, TNF in supernatants were measured by ELISA ( $n=3$ replicate wells). $\mathbf{b}$ Zc3h12a expression in WT BMDMs was measured by qPCR ( $n=5$ mice per group). c Zc3h12a expression in IMQ-treated skin of WT mice at indicated days was measured by qPCR ( $n=3$ mice per group). $\mathbf{d}$ Degradation of Zc3h12c mRNA by Regnase-1 was measured by a luciferase reporter system. Zc3h12c 3'UTR (separated into three segments) were inserted downstream of the luciferase gene ( $n=6$ replicates). e Zc3h12a expression in $Z c 3 h 12 c^{+/} / Z c 3 h 12 c^{-/}-$FL3TL-pDCs and BMDMs was measured by qPCR $(n=4$ mice per group). $\mathbf{f}$ Nfkbiz expression in Zc3h12c $c^{+/+} / Z c 3 h 12 c^{-/-}$FL3TL-pDCs was measured by qPCR ( $n=4$ mice per group). $\mathbf{g}$ Degradation of $N f k b i z$ mRNA by MCPIP3 was measured by a luciferase reporter system ( $n=6$ replicates). h-j Expression of indicated NFKB family members in Zc3h12c $\mathrm{c}^{+/+} / \mathrm{Zc} 3 \mathrm{~h} 12 \mathrm{c}^{-/-} \mathrm{FL} 3 \mathrm{TL}-\mathrm{pDCs}$ was measured by qPCR ( $n=4$ mice per group). Data are representative of three (a-e) or two (f-j) independent experiments (mean \pm S.D.; two-tailed Student's $t$ test). Source data are provided as a Source Data file.

binding to Zc3h12c $3^{\prime}$ UTR (Fig. 8d). In comparison, Regnase-1 expression in naïve or activated pDCs was minimal $(\sim 15$-fold less) when compared to activated macrophages (Fig. 8e). Here we demonstrate that Regnase-1 could degrade MCPIP3 in macrophages, but is little expressed in pDCs.

Besides cytokines and family members, we screened the mRNA expression levels of transcriptional regulators such as A20, HuR, TTP (ZFP36), IRF3/7, and NFKB signaling pathway molecules in $Z c 3 h 12 c^{-1-}$ macrophages and pDCs (Supplementary Figs. 6, 7). We found little difference in macrophages, possibly because of the transient expression of MCPIP3 and compensation by Regnase-1 (Supplementary Fig. 6a). Thus, we focused on pDCs, because they expressed the highest level of MCPIP3, and little Regnase-1. We

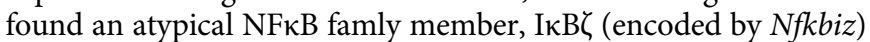
was upregulated in MCPIP3-deficient pDCs at early and late timepoints of activation (Fig. 8f). To determine if Nfkbiz is a direct degradative target of MCPIP3, we utilized the same luciferase reporter system. MCPIP3 could significantly decrease luciferase signals linked to Nfkbiz 3'UTR, at an even better efficiency than Regnase-1 (Fig. 8g). In addition, expression of several members of the NFKB family were affected by MCPIP3deficiency in pDCs (Fig. 8h-j, Supplementary Fig. 7b). However, most of these changes occurred at late phase, rather than early phase after activation (Fig. $8 \mathrm{~h}-\mathrm{j}$ ). Besides R848, we also found similar results with CpG B activation. (Supplementary Fig. 7c). In contrast, MCPIP3 did not seem to regulate c-Rel expression, another known target of Regnase-1 (Fig. 8j). Here we conclude that MCPIP3 could regulate NFkB pathway by directly degrading $\mathrm{I} \kappa \mathrm{B} \zeta$ mRNA.

\section{Discussion}

Herein, we found that MCPIP3 promotes psoriatic skin inflammation by differentially regulating TNF, IL-6 and IL-12p40 secretion by macrophages and pDCs. In macrophages, TNF regulation during early-phase and late-phase is quite distinct, and is differentially regulated by I $\mathrm{BB} \alpha$ and $\mathrm{A} 20^{59}$. Similarly, Regnase- 1 and roquin 1 promote the decay of inflammatory mRNAs at different phases of the inflammatory response ${ }^{14}$. Regnase- 1 controls the early phase of inflammation via the cleavage and degradation of translationally active mRNAs, whereas roquin 1 controls the late phase of inflammation by removing translationally inactive mRNAs ${ }^{14}$. Our observations are consistent with a previous report which found no difference in MCPIP3-deficient macrophage TNF secretion after $8 \mathrm{~h}$ (late-phase). Unfortunately, this report did not examine any early timepoints ${ }^{24}$. Collectively, our results suggest early-phase of macrophage activation could be a key to initiate autoimmune skin inflammation. However, a defect in macrophage TNF could not fully account for the phenotype in MCPIP3-deficient mice.

Although the pro-inflammatory roles of TNF and IL-12 are well-established, the immunosuppressive role of IL-6 was overlooked in autoimmunity. IL- 6 could switch between inflammatory and anti-inflammatory roles, depending on specific scenarios $^{2}$. Mechanistically, IL-6 signaling via the soluble IL-6R (trans-signaling) is pro-inflammatory, whereas classic signaling via the membrane-bound receptor is regenerative and antiinflammatory ${ }^{60}$. In psoriasis, IL-6 has been long associated with disease pathogenesis ${ }^{61}$. IL- 6 is produced by keratinocytes, fibroblasts, endothelial cells, dendritic cells, macrophages, and Thelper type 17 cells $^{27,61}$. However, genetic deletion of IL-6 can worsen psoriasis in certain animal models ${ }^{62}$. Clinically, IL-6 targeted blockade are ineffective or even detrimental in psoriasis and other indications ${ }^{6}$. Anti-IL-6 receptor antibody tocilizumab has been reported to induce a psoriasis-like disease in some patients $^{63}$. Thus, we hypothesize that IL-6 may play differential roles during early and late stages of psoriasis pathogenesis. IL-6 may be anti-inflammatory during early phase of psoriasis, when pDCs were heavily recruited. During late phase, IL-6 stimulated proliferation of cultured human keratinocytes, a major hallmark of psoriatic lesions ${ }^{61}$. Skin inflammation was also abrogated in IL6-deficient mice with recombinant IL-23-induced psoriasiform lesions ${ }^{64}$. IL-6 may synergize with IL-17C and TNF to drive a psoriasis-like signature from keratinocytes ${ }^{62}$. Therefore, IL-6 could be pro-inflammatory during late phase of psoriasis pathogenesis.

While the aberrant production of IFN- $\alpha$ by pDCs clearly promotes IFN-I-driven autoimmunity such as systemic lupus erythematosus, the exact role of pDCs in psoriasis is still puzzling and controversial ${ }^{50}$. In addition, pDC-derived IFN- $\alpha$ was reported to be involved in pro-inflammatory processes and promote wound healing ${ }^{65-67}$. However, genetic ablation of pDCs or IFN-I receptors did not affect the severity of imiquimod-induced psoriatic plaque formation in mice ${ }^{46,47}$. It is puzzling that activated pDCs were recruited to inflamed skin, but played nonessential roles in psoriasis. Because IFN-I was long-seen as the exclusive functional cytokine secreted by pDCs, most studies focused only on IFN-I signaling. pDC-derived IL-6 may offer a plausible mechanism to explain pDC's puzzling role in psoriasis.

The conclusion that MCPIP3 may promote skin inflammation is unusual. However, our characterization of MCPIP3 protein function per se is quite similar to its family members. First, TPP, Roquin and Regnase- 1 are potent degraders of IL- 6 mRNA ${ }^{8}$. We found that MCPIP3 could also degrade IL- 6 mRNAs. Second, Regnase-1 is known to regulate NFKB pathway, and we found MCPIP3 could directly degrade $I \kappa B \zeta^{14,20}$. Third, Regnase- 1 was previously proposed to be MCPIP3's degradative target, and we found similar results ${ }^{24}$. Nevertheless, the physiological function of the RNase is dependent on its degradative targets. TNF and IL$12 \mathrm{p} 40$ are known degradative target of Regnase- $1^{15}$. IкB $\zeta$ deficient mice showed severe skin irratation, and $I \kappa B \zeta$ is reported to promote IL-6 expression, while suppressing TNF ${ }^{68-70}$. Therefore, MCPIP3 may promote TNF/IL-12 via Regnase-1, and suppress IL-6 via direct mRNA degradation and indirectly via $\mathrm{I} \kappa \mathrm{B} \zeta$.

Our study does not fully explain how MCPIP3 expression is dynamically regulated, or how does MCPIP3 achieve its degradative specificity. First, the spatiotemporal expression of MCPIP3/Regnase-1 in macrophages and pDCs seemed to be 
designed for maximum synergy to promote inflammation. The rapid degradation of MCPIP3 by Regnase-1 might explain why MCPIP is dispensable for late phase TNF secretion in macrophages. Similarly, the lack of Regnase-1 might explain how MCPIP3 is dispensable for TNF production in pDCs. Besides Regnase-1, a recent report found that macrophages upregulated MCPIP3 upon TLR3 agonist Poly:IC stimulation, and MCPIP3 expression was dependent on IRF3/7 but not $\mathrm{NFKB}^{24}$. We found robust MCPIP3 upregulation after activation by TLR4/7/9 ligands in macrophages and pDCs. Our results were consistent because TLR7/9 signaling signals were dependent on IRF3/739. Second, additional binding partners may be required to increase MCPIP3 specificity to its degradative targets, as mRNA $3^{\prime}$ UTRs are not unique enough to allow the precise recognition of individual mRNAs. For example, Regnase-1 requires the helicase activity of UPF1, as well as roquin 1 for the efficient degradation of IL-6 mRNA ${ }^{14}$. We have consistently shown that MCPIP3 could bind and degrade IL-6, Regnase-1, or IкB $\zeta$ mRNA by their $3^{\prime}$ UTRs, but not other negative control genes. However, a previous report concludes that MCPIP3's mRNA degradative activity is unspecific, as MCPIP3 degraded all their samples including negative controls ${ }^{24}$. This experimental discrepancy could be due to the presence of unknown co-factors that facilitate or specify MCPIP3's recognition of mRNAs.

Overall, our findings demonstrate that RNase MCPIP3 orchestrates TNF and IL- 6 secretion by macrophages and pDCs to exert a surprisingly pro-inflammatory role in psoriatic lesions. This suggests that MCPIP3 could be a potential inhibitory target to treat psoriasis and other autoimmune diseases.

\section{Methods}

Human samples. Blood sample collection from healthy donors: Peripheral blood samples were collected from healthy donors, after taking written informed consent, in accordance with the Declaration of Helsinki and as recommended and approved by the institutional review board of CSIR-Indian Institute of Chemical Biology, Kolkata, India.

Immune cell subset isolation: PBMC were isolated from whole blood by sucrose density-gradient centrifugation using Hisep LSM (Ficoll). The various immune cell subsets $\left(\mathrm{B}\right.$ cells, $\mathrm{CD} 4^{+} \mathrm{T}$ cells, $\mathrm{CD} 8^{+} \mathrm{T}$ cells, monocytes, $\left.\mathrm{CD} 1 \mathrm{c}^{+} \mathrm{cDCs}, \mathrm{pDCs}\right)$ were isolated by magnetic immune-selection using CD19, CD4, CD8, CD14, CD1c, and BDCA4 microbeads (Miltenyi Biotec) respectively. For monocyte-derived DC $(\mathrm{moDC})$ generation, $\mathrm{CD} 14^{+}$monocytes were cultured for 5 days at a density of 0.5 million cells per well, in the presence of GM-CSF $(20 \mathrm{ng} / \mathrm{ml})$ and IL- $4(10 \mathrm{ng} / \mathrm{ml})$. For monocyte-derived macrophages (M0 macrophage) generation, CD14 ${ }^{+}$ monocytes were seeded at a density of 0.25 million cells per well (48-well plate), cultured for 7 days in the presence of M-CSF $(20 \mathrm{ng} / \mathrm{ml})$. After 7 days, M0 macrophage were treated with IFN- $\gamma(20 \mathrm{ng} / \mathrm{ml})$ and LPS $(100 \mathrm{ng} / \mathrm{ml})$ to generate M1 macrophage; or with IL-4 $(20 \mathrm{ng} / \mathrm{ml})$ to generate M2 macrophages.

Gene expression assays: Total RNA was isolated from the immune cell subsets using TRIzol reagent (Invitrogen, USA) and cDNA prepared (MultiScribe Reverse Transcriptase cDNA kit, Invitrogen) following manufacturer's protocol. Quantitative PCR was carried out (QuantStudio 3 PCR machine) for Zc $3 h 12 c$ gene, with each sample being run in triplicates and normalized against $18 \mathrm{~S}$ rRNA as the housekeeping gene.

Transgenic mice. C57BL/6J (WT), B6.SJL-Ptprc ${ }^{a} P e p c^{b} / B o y J\left(C D 45.1^{+}\right)$, B6.129S2Ifnar1 ${ }^{\mathrm{tm} 1 \mathrm{Agt} / \mathrm{Mmjax}}\left(\right.$ Ifnarl $\left.^{-1-}\right)$, B6.129P2-Lyz2tm1 (cre) $)^{\mathrm{Ifo} / \mathrm{J}}$ ( Lyz2-Cre) and B6.Cg$\operatorname{Tg}(\text { Itgax }- \text {-cre })^{1-1 R e i z / J}($ Itgax-Cre $)$ were purchased from The Jackson Laboratory. $Z c 3 h 12 c^{-l-}$ and $Z c 3 h 12 c^{\mathrm{fl} / \mathrm{ll}}$ mice were generated in-house and could be provided upon reasonable request. $Z c 3 h 12 c^{\mathrm{fl} / \mathrm{fl}}$ mice were generated with technical expertise from GemPharmatech Co. Ltd. Age and sex-matched mice between 6 and 10 weeks of age were used. All mice were bred and maintained in specific-pathogen free (SPF) at GemPharmatech Co. Ltd. and BSL3 facilities at SYSU. This study complied with all relevant ethical regulations for animal testing research according to the institutional guidelines and protocols was approved by the Animal Ethics Committee of Sun Yat-sen University.

Generation of $Z c 3 h 12 c^{-1-}$ germline knockout mice: In short, Cas9 mRNA and CRISPR-Cas9 sgRNA were co-injected into zygotes. -1 bp indel frame shift mutation was obtained on exon 3, resulting in a premature stop codon on exon 4 . Mice were genotyped by sequencing PCR products amplified with the following primers (Zc3h12c-Fwd: ACT TGA ATT TGG GTT TCA TTG TGC T, Zc3h12cRvs: TCC CTA GAG TGA CAA GGC CC). To avoid any off-target mutations, the resulting mice were backcrossed with $\mathrm{C} 57 \mathrm{BL} / 6 \mathrm{~J}$ for at least five generations, and the top predicted ten CRISPR-Cas9 off-target sites were sequenced and verified to be unmutated. Whole exon sequencing was also performed to ensure the only $Z c 3 h 12 c$ was targeted.

Generation of $Z c 3 h 12 c^{\mathrm{fl} / \mathrm{fl}}$ conditional knockout mice: Cas9 mRNA and sgRNA were co-injected into zygotes. sgRNA directed Cas9 endonuclease cleavage at intron 2-3 and intron 3-4, and created a DSB (double-strand break). Such DSBs would be repaired, and result in loxP sites inserted into intron 2-3 and intron 3-4 respectively by homologous recombination. Mice were genotyped via PCR (Zc3h12c-fl-Fwd: ACC AGG TTA GTG CTA ACA GTG G, Zc3h12c-fl-Rvs: CAC TGC ATG GCC TAC CCT GA). The insertion of loxP sites were confirmed by expected PCR bands of $217 \mathrm{bp}$, as the wildtype band was $122 \mathrm{bp}$. Cell-specific deletion of $Z c 3 h 12 c$ allele was obtained after Cre-mediated recombination after crossed with Lyz2-Cre or Itgax-Cre. Deletion in both Cre lines were confirmed PCR and QPCR with FACS-sorted macrophages or dendritic cells.

Generation of chimeras. $\mathrm{CD} 45.1^{+}$or $Z c 3 h 12 c^{-1-}$ mice were lethally irradiated with $950 \mathrm{Rad}$ and then injected i.v. with $5 \times 10^{6}$ bone marrow cells harvested from age and sex-matched CD45.1 $1^{+}$or $Z c 3 h 12 c^{-1-}$ mice. The mice were fed with $0.5 \mathrm{~g} / \mathrm{L}$ neomycin sulfate hydrate (BBI life sciences, Cat\#A610366-0100) for 2 weeks and then rested for at least an additional 4 weeks to allow reconstitution of immune cells.

Induction of psoriasiform lesions. IMQ: Mice between 8 and 12 weeks of age received a daily topical dose of $62.5 \mathrm{mg}(3.125 \mathrm{mg}$ of the active compound) of Imiquimod (IMQ) cream (5\%; Aldara; $3 \mathrm{M}$ Pharmaceuticals) on the shaved back or ears for consecutive days. Aldara (IMQ) cream (Pharmaceutical grade) was obtained from iNova (3M Health Care Limited). Back-skin samples were excised using $5 \mathrm{~mm}$ biopsy punch and extracted for lymphocytes. Skin punches were also flash frozen, and stored at liquid nitrogen before isolation for total RNA. To score the severity of inflammation of the back skin, an objective scoring system was developed based on the clinical Psoriasis Area and Severity Index (PASI), except that for the mouse model the affected skin area is not taken into account in the overall score (mPASI). Erythema and scaling were scored independently on a scale from 0 to 4: 0 , none; 1 , slight; 2 , moderate; 3 , marked; or 4 , very marked. The level of erythema was scored using a scoring table with red taints. The cumulative score (erythema plus scaling) served as a measure of the severity of inflammation (scale 0-8). All measurements were performed blinded, without knowledge of mice genotype.

Recombinant IL-23: mice ears were intradermally injected with $20 \mu$ of vehicle (PBS) or $500 \mathrm{ng}$ of recombinant mouse IL-23 (Cat\# CT028-M08H,Sino Biological) using a 33-gauge needle. Injections were repeated on alternate days for a total of five to seven doses. Ear thickness was measured on days without injection with 0.01-mm-thickness gauge dial micrometer (TECLOCK JAPAN). All measurements were performed blinded, without knowledge of mice genotype. Mice were sacrificed and skin samples were collected using the same procedures as IMQ-treated mice.

Recombinant IL-6: mice back skins were intradermally injected with $100 \mu \mathrm{l}$ vehicle (PBS) or $2 \mathrm{ug} / 200 \mathrm{ng} / 20 \mathrm{ng} / 2 \mathrm{ng}$ (in 100ul) of recombinant mouse IL-6 (Cat\# 50136-MNAE, Sino Biological) for 4 days, starting from the day of IMQ application.

Wound healing and septic shock. Skin healing: Full-thickness wounds were created by excision of 4-mm punches on shaven back skin. Prior to the surgery, aged-matched mice were anesthetized with isoflurane and subcutaneous injections of buprenorphine $(0.5 \mathrm{mg} / \mathrm{kg})$ were given as analgesics. Wound area was measured by tracing onto a glass slide at the indicated timepoints. Slides were scanned and area calculated with ImageJ software.

Septic shock: LPS from E. coli 055:B5 (Cat\#L12880,Sigma-Aldrich) and D(+)-Galactosamine hydrochloride(CAS\# 1772-03-8, Macklin) were diluted in pyrogen-free saline. A combination of LPS ( $5 \mu \mathrm{g}$ per kg body weight) and D-gal (400 mg per kg body weight) was injected intraperitoneally. The animal's survival was scored for mortality between 0 and $24 \mathrm{~h}$

EAE induction. For active EAE induction, age- and sex-matched mice were immunized s.c. with $\mathrm{MOG}_{35-55}$ (Genscript, purity >98\%) peptide (300 $\mu \mathrm{g}$ ) emulsified in CFA containing $4 \mathrm{mg} / \mathrm{ml}$ heat-killed MTB (Cat\#7001,Chondrex). $200 \mathrm{ng}$ Pertussis toxin (Cat\#181239A1, List Biological Laboratories) dissolved in PBS was administered i.p. on days 0 and 2. Mice were examined daily and scored for disease severity using the standard scale: 0 , no clinical signs; 1 , limp tail; 2 , paraparesis (weakness, incomplete paralysis of one or two hind limbs); 3, paraplegia (complete paralysis of two hind limbs); 4 , paraplegia with forelimb weakness or paralysis; 5 , moribund or death. After the onset of EAE, food and water were provided on the cage floor. Mononuclear cells were prepared from the CNS (brain and spinal cord) of EAE-induced mice as described and analyzed by flow cytometry.

In vivo infections. For LM-OVA infection, LM-OVA was grown in TSB medium to an $\mathrm{OD}_{600}$ of $\sim 0.25$, diluted in PBS and injected $\left(8 \times 10^{3} \mathrm{CFU}\right)$ in a volume of 0.2 $\mathrm{ml}$ per mouse i.v.. After 5 days, the mice were analyzed. For the LM-CFU colony forming assay, $100-\mu \mathrm{l}$ samples were added to TSB solid medium and incubated overnight at $37^{\circ} \mathrm{C}$. For LCMV clone 13 infection, $1 \times 10^{6} \mathrm{PFU}$ were diluted in PBS 
and injected i.p. into mice. LCMV titers were determined by extracting mRNA from extracted organs, and performing qPCR with virus-specific primers.

Immune cell isolation. Skin: Mouse ear or back skin was incubated in $0.25 \%$ Trypsin-EDTA (25-050-CI, Corning) for $45 \mathrm{~min}$, and epidermis and dermis layers were separated with forceps. Separated skin layers were chopped into small pieces and incubated in digestion buffer $(1 \mathrm{mg} / \mathrm{mL}$ Collagenase P, $0.1 \mathrm{mg} / \mathrm{mL}$ DNase I, and $1 \mathrm{mg} / \mathrm{mL}$ Dispase II) for 45 to $90 \mathrm{~min}$, respectively, to obtain single-cell suspension.

Lymphoid organs: To harvest immune cells from lymphoid tissue, organs were minced, ground up, and passed through a 70- $\mu \mathrm{m}$ nylon mesh. Erythrocytes were removed using ACK lysis buffer ( $150 \mathrm{mM}$ ammonium chloride, $10 \mathrm{mM}$ potassium bicarbonate, and 0.1 mM EDTA). The cells were counted using Beckman Coulter CytoFlex. Before sorting, DCs were enriched with CD11c microbeads (Miltenyi Biotec). For peripheral tissues, organs were digested in collagenase D (Roche) and DNase I (Sigma-Aldrich) for $1 \mathrm{~h}$ at $37^{\circ} \mathrm{C}$ with stirring in PBS. After enzymatic treatment, tissues were further dissociated over a $70 \mu \mathrm{m}$ nylon cell strainer. For isolation of lymphocytes single-cell suspensions were then separated using a $44 \%$ / 67\% Percoll (Sigma) density gradient.

Peritoneal cavity macrophages: Peritoneal exudate cells (PECs) were obtained by lavaging the peritoneal cavity twice with $5 \mathrm{~mL}$ ice-cold PBS. PECs were collected in a $15-\mathrm{ml}$ tube, and centrifuge at $500 \times g$ for $5 \mathrm{~min}$. Peritoneal macrophages were purified from PECs $(>90 \%)$ with short incubating on tissue-culture plates and washing away unattached cells. These cells were stimulated by various agonists as indicated.

In vitro stimulation and flow cytometry. In case of intracellular cytokine staining, 10ug/ml brefeldin A (BFA, eBiosciences) was added with TLR ligands $(1 \mu \mathrm{g} / \mathrm{ml}$ R848, $1 \mu \mathrm{M}$ CpG A, $1 \mu \mathrm{M}$ CpG B, $1 \mu \mathrm{g} / \mathrm{mL}$ Pam3CSK4, 100 ng/mL LPS, $500 \mathrm{ng} / \mathrm{mL}$ Poly(I:C) for 4-16h before staining with the intracellular staining kit (Cat\#888824-00, Thermofisher). In case of ELISA, supernatants were collected 4-16 h after TLR stimulations. The following antibodies were all used at dilution of 1:400 (unless otherwise indicated):

eBioscience CD45FITCClone:30-F11Catalogue \#:11-0451-82 eBioscience CD45PEClone:30-F11Catalogue \#:12-0451-82 eBioscience CD45APCClone:30-F11Catalogue \#:17-0451-82 eBioscience CD45.1PE-Cyanine7Clone:A20Catalogue \#:25-0453-82 eBioscience CD45.1PBClone:A20Catalogue \#:48-0453-82 eBioscience CD45.2APC-Cyanine7Clone:104Catalogue \#:47-0454-82 Biolegend XCR1PerCP/Cyanine5.5Clone: ZETCatalogue \#:148207 eBioscience CD172aPEClone:P84Catalogue \#:12-1721-82 eBioscience CD172aPE-Cyanine7Clone:P84Catalogue \#:25-1721-82 eBioscience MHC Class IIPEClone:M5/114.15.2Catalogue \#:12-5321-82 eBioscience MHC Class IIPE-Cyanine7Clone:M5/114.15.2Catalogue \#: 25-5321-82 eBioscience MHC Class IIPBClone:M5/114.15.2Catalogue \#:48-5321-82 eBioscience CD11cPEClone:N418Catalogue \#:12-0114-82

eBioscience CD11cAPC-Cyanine7Clone:N418Catalogue \#: 47-0114-82 eBioscience CD8aPerCP/Cyanine5.5Clone:53-6.7Catalogue \#:35-0081-82 eBioscience CD11bPerCP-Cyanine5.5Clone:M1/70Catalogue \#:45-0112-82 eBioscience CD11bFITCClone:M1/70Catalogue \#:11-0112-82 eBioscience CD4APC-Cyanine7Clone:RM4-5Catalogue \#: 47-0042-82 eBioscience CD4eFluor 450Clone:RM4-5Catalogue \#:48-0042-82 eBioscience BST2APCClone:eBio927Catalogue \#:17-3172-82 eBioscience BST2FITCClone:eBio927Catalogue \#: 11-3172-82 eBioscience SIGLEC HAPCClone:eBio440cCatalogue \#:17-0333-82 eBioscience SIGLEC HFITCClone:eBio440cCatalogue \#:11-0333-82 eBioscience B220eFluor 450Clone:RA3-6B2Catalogue \#:48-0452-82 eBioscience TNF alpha PE-Cyanine7 Clone:MP6-XT22Catalogue \#:25-7321-82 eBioscience CD24PEClone:M1/69Catalogue \#:12-0242-82 eBioscience F4/80PEClone:BM8Catalogue \#:12-4801-82 eBioscience F4/80eFluor 450Clone:BM8Catalogue \#:MF48004-3 eBioscience IgDAPC-eFluor 780Clone:11-26c(11-26)Catalog \#:47-5993-82 eBioscience IgM PEClone:II/41Catalogue \#:12-5790-82

eBioscience IgM Super Bright 600Clone:II/41Catalogue \#:63-5790-82 eBioscience CD93APCClone:AA4.1Catalogue \#:17-5892-82 Biolegend CD5APCClone:53-7.3Catalogue \#:100626

Biolegend Ly-6CAlexa Fluor ${ }^{\circledR}$ 488Clone:HK1.4Catalogue \#:128022 eBioscience Ly-6GeFluor 450Clone:1A8-Ly6gCatalogue \#:48-9668-82 eBioscience CD3e APCClone:145-2C11Catalogue \#:MA1-10186 eBioscience CD3e eFluor 450Clone:145-2C11Catalogue \#:48-0031-82 eBioscience TCR- $\gamma \delta$ APCClone:eBioGL3Catalogue \#:17-5711-82 eBioscience TCR- $\gamma \delta$ PE-Cyanine7 Clone:eBioGL3 Catalog \#:25-5711-82 eBioscience TCR $\beta$ PEClone:H57-597Catalogue \#:12-5961-82 eBioscience TCR $\beta$ APC-eFluor 780Clone:H57-597Catalogue \#:47-5961-82 eBioscience IFN- $\gamma$ PEClone:XMG1.2Catalogue \#:12-7311-82 eBioscience IFN- $\gamma$ PerCP/Cyanine5.5Clone:XMG1.2Catalogue \#:45-7311-82 eBioscience IL-17AFITCClone:eBio17B7Catalogue \#:11-7177-81 Biolegend CD80FITCClone:16-10A1Catalogue \#:104706 Biolegend CD80PEClone:16-10A1Catalogue \#:104708 MBL Ly49QPEClone:2E/6Catalogue \#: D160-4 eBioscience CD86FITCClone:GL1 Catalog \#:11-0862-82
eBioscience CD86PEClone:GL1 Catalog \#:12-0862-82

eBioscience CD199PerCP-eFluor 710Clone:eBioCW-1.2Catalogue \#:46-1991-82 eBioscience CD64PerCP-eFluor 710Clone:X54-5/7.1Catalogue \#:46-0641-82 PBLassay IFNaFITCClone:RMMA-1Catalogue \#:22100-3 Biolegend CCR2PE-Cyanine7Clone:QA18A56Catalogue \#:160108 eBioscience Ly-6A/E (Sca-1)APCClone:D7Catalogue \#: 17-5981-82 eBioscience Ly-6A/E (Sca-1)PE-Cyanine7Clone:D7Catalogue \#:25-5981-82 eBioscience IL-12p40eFluor 660Clone:C17.8Catalogue \#:50-7123-82 Invivogen IL-6N.AClone:10F9Catalogue \#:mabg-mil6-3

Flow cytometry was performed on Beckman Coulter CytoFlex and analyzed using FlowJo software (Tree Star, version X). MFI were calculated by genomic mean in FlowJo. Cell-sorting experiments were conducted on a BD FACSAria III. Staining was performed at $4^{\circ} \mathrm{C}$ in the presence of Fc block (Clone 2.4G2; BD) and FACS buffer (PBS, $0.5 \%$ BSA, 2 mM EDTA, 0.1\% sodium azide).

Primary cell culture. Mice were sacrificed by cervical dislocation and removal of both femurs and tibias. After removing the muscle and fat with a pair of scissors, the femurs and tibias were put in $5 \mathrm{ml}$ RPMI 1640 (10\% FCS and PS) on ice. The ends of the bone were cut with a sharp pair of scissors in the tissue-culture hood and then the BM were flushed out with a 10-ml syringe (25-G needle) and RPMI 1640 media $(10 \%$ FCS, PS). BM cells were collected in a $15-\mathrm{ml}$ tube, and centrifuge at $500 \times g$ for $5 \mathrm{~min}$.

Bone marrow-derived macrophages (BMDM). $5 \times 10^{6}$ bone marrow cells per well were cultured in non-pyrogenic sterilized $90 \mathrm{~mm}$ petri dishes in RP10 (RPMI supplemented with glutamine, penicillin, streptomycin; Invitrogen), 10\% FBS and $30 \%$ supernatants of L929 mouse fibroblasts (ATCC ${ }^{*}$ CRL-6364 $4^{\mathrm{m}}$ ) as conditioned medium providing macrophage colony stimulating factor (M-CSF). Half of media was replenished on day 3 and day 6 , and harvested on day 7 . To harvest macrophages, cultured cells were washed once with ice-cold PBS, and then incubated in PBS containing $20 \mathrm{mM}$ EDTA and $20 \%$ FCS at $37^{\circ} \mathrm{C}$ for $5 \mathrm{~min}$. BMDMs were gated as live ${ }^{+} \mathrm{CD}_{4} 5^{+} \mathrm{CD}_{11 \mathrm{~b}}{ }^{+} \mathrm{F} 4 / 80^{+}$cells.

FL3TL-pDCs. $4 \times 10^{6}$ bone marrow cells per well were cultured in tissue-culturetreated 6-well plates in $4 \mathrm{ml}$ of RP10 (RPMI 1640 supplemented with glutamine, penicillin, streptomycin, 2-mercaptoethanol [all from Invitrogen]), 10\% heatinactivated fetal calf serum (Source BioSciences), and 30\% supernatants of Flt3LB16 cell line (ATCC ${ }^{\circledR}$ CRL- $6475^{\mathrm{Tw}}$ retrovirally transduced in house with murine Flt3l) as conditioned medium providing Flt3L. FL3TL-pDCs were gated as live ${ }^{+}$ $\mathrm{CD}_{11 \mathrm{c}^{+} \mathrm{B}^{2} 20^{+} \text {SiglecH }}+$ cells. These cells were harvested on day 7 .

BMDC. $4 \times 10^{6}$ bone marrow cells per well were cultured in tissue-culture-treated 6-well plates in $4 \mathrm{ml}$ of RP10 (RPMI 1640 supplemented with glutamine, penicillin, streptomycin, 2-mercaptoethanol [all from Invitrogen]), 10\% heat-inactivated fetal calf serum (Source BioSciences), IL-4 $(5 \mathrm{ng} / \mathrm{ml}$, Peprotech) and GM-CSF $(20 \mathrm{ng} / \mathrm{ml}$ Peprotech). Half of the medium was removed at day 2 , and new medium supplemented with GM-CSF $(40 \mathrm{ng} / \mathrm{ml}), \mathrm{IL}-4(10 \mathrm{ng} / \mathrm{ml})$ which were pre-warmed at $37^{\circ} \mathrm{C}$ was added. The culture medium was entirely discarded at day 3 and replaced with fresh warmed medium containing GM-CSF $(20 \mathrm{ng} / \mathrm{ml})$ and IL- $4(5 \mathrm{ng} / \mathrm{ml})$. BMDCs were gated as live ${ }^{+} \mathrm{CD}_{11 \mathrm{~b}^{+}} \mathrm{MHCII}^{+} \mathrm{CD} 11 \mathrm{c}^{+}$cells. These cells were harvested on day 6 .

$p D C s / B M D M$ co-culture. $5 \times 10^{4}$ Flt3L-pDCs were plated on 96-well plates with varying numbers, and pre-condition with $1 \mu \mathrm{g} / \mathrm{mL}$ R 848 for $16 \mathrm{~h}$. Varying ratio of BMDMs were added in wells containing Flt3L-pDCs as indicated in the figure legends. These co-cultured cells were stimulated for $4 \mathrm{~h}$ with $1 \mu \mathrm{g} / \mathrm{mL}$ R848 in presence of $10 \mu \mathrm{g} / \mathrm{mL}$ Brefeldin A for intracellular cytokines stain. pDC supernatant with BMDM: BMDM were stimulated with $1 \mu \mathrm{g} / \mathrm{mL}$ R848 in presence of $10 \mu \mathrm{g} / \mathrm{mL}$ Brefeldin A in medium containing various concentration of supernatant from Flt3L-pDCs which were stimulated for $16 \mathrm{~h}$ with indicated ligands.

Cytokine/antibodies additions. rIL6: BMDM were treated with IL-6 (Cat\#50136MNAE, Sino Biological) for along with $1 \mu \mathrm{g} / \mathrm{mL}$ R848 for $4 \mathrm{~h}$. IFN-I: BMDMs were pre-conditioned with IFN-alpha4 (Cat\#50672-M08H, Sino Biological) for 16, 24, or $48 \mathrm{~h}$, and then activated with $\mathrm{R} 848(1 \mu \mathrm{g} / \mathrm{ml})$ for $4 \mathrm{~h}$. The supernatant was harvested and stored in $-80^{\circ} \mathrm{C}$ refrigerator for subsequent ELISA measurement. Anti-IL-6: $3.5 \times 10^{5}$ sorted (purity $>99 \%$ ) Flt3L-pDC activated with $1 \mu \mathrm{g} / \mathrm{ml}$ R848 containing in $1.4 \mathrm{ml} \mathrm{RP10}$ for $24 \mathrm{~h}$. Then Flt3L-pDC supernatants were pre-incubated with $1 \mu \mathrm{g} / \mathrm{ml}$ anti-IL6 mIgG (Cat\#mabg-mIL6-3, Invivogen) for $30 \mathrm{~min} .3 \times 10^{5} \mathrm{BMDMs}$ were co-cultured with 50\% Flt3l-pDC supernatant or anti-IL6 pre-treated Flt3L$\mathrm{pDC}$ supernatant in the presence of $1 \mu \mathrm{g} / \mathrm{ml} \mathrm{R} 848$ and $10 \mu \mathrm{g} / \mathrm{ml}$ brefeldin A for $4 \mathrm{~h}$, and were analyzed by intracellular staining with FACS.

Molecular cloning. Mouse cDNA of Zc3h12a (NM_153159.2), Zc3h12c (NM_001162921.2), Il6 (NM_031168.2), Tnf (NM_013693.3), Nfkbiz(NM_030612) were reverse transcribed with HiScript II 1st Strand cDNA Synthesis Kit (Vazyme, Cat\#R212-02) from the mRNA of BMDM after treated with R848 for $4 \mathrm{~h}$, then al were cloned into pTOPO vector (GenStar,Cat\#T182-20). Point mutations of the 
gene encoding Zc3h12a (D141N, C316R) and Zc3h12c (D271A, D271N, D271A, D356A, S372A, D374A, D378A, C436R), and human SNP rs4561177 were made with Mut Express II Fast Mutagenesis Kit V2 (Vazyme, Cat\#C214-02). Myc-tag and Flag-tag were added to the N-terminal of CDS of Zc3h12a and Zc3h12c respectively. The CDSs of N-Myc-Zc3h12a and N-Flag-Zc3h12c were ligated to the vector pWPI or pLVX for expression. Full length of 3'UTR of Il6 (1-427), Tnf (1764), $\beta$-actin (1-683), Zc3h12a (1-856), Zc3h12c (1-3794), ccr7 (1-762), Nfkbiz (11294) were insert into the pGL3 vector at the site of XbaI site. These CDS + $3^{\prime} \mathrm{UTR}$ was inserted into pLVX-puro (Clontech, Cat \# 632164) or pWPI (Addgene, Cat\#12254), along with pGL3 vector.

Luciferase reporter assay. 293T (ATCC ${ }^{\circ}$ CRL-3216 ${ }^{\mathrm{mm}}$ ) cells were transfected with the firefly/Renilla DuoLuc-Luciferase reporter vector and an expression plasmid for as indicated via Exfect 2000 Transfection Reagent (Vazyme, Cat\# T202-02). $48 \mathrm{~h}$ later, the cells were lysed, and firefly and Renilla activity was determined according to the manufacturer's protocol of the Dual Luciferase Reporter Assay Kit (Vazyme, Cat\#DL101-01). Firefly activity was normalized to the Renilla activity.

Protein degradation assay. For Regnase- 1 degradation assay, $2.5 \times 10^{5} 293 \mathrm{~T}$ cells

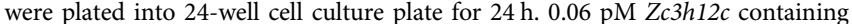
pLVX-vector were co-transfected with $0.01 \mathrm{pM} Z \mathrm{Zc} 3 \mathrm{~h} 12 a$-CDS or Zc3h12a-CDS-3' UTR containing pWPI-plasmid into 293T cell in the presence of PEI. After $48 \mathrm{~h}$, whole cell extracts were prepared with low-salt lysis buffer (50 mM HEPES, $150 \mathrm{mM} \mathrm{NaCl}, 1 \mathrm{mM}$ EDTA, $10 \%$ glycerol, $1.5 \mathrm{mM} \mathrm{MgCl}$, and 1\% Triton X-100) after transfection. The samples were denatured with $5 \times$ loading buffer (Genstar, E153-05) for $5 \mathrm{~min}$ at $95^{\circ} \mathrm{C}$. Total cell lysates $(60 \mu \mathrm{g})$ were running on SDS-PAGE with $4-12 \%$ gradient gel and then transferred. Proteins separated by SDS-PAGE were transferred to polyvinylidene fluoride (PVDF) membranes (Bio-Rad). The membranes were blocked by $5 \%$ dried milk at $4{ }^{\circ} \mathrm{C}$ overnight, and then incubated with HRP-conjugated antibodies for $1 \mathrm{~h}$ at room temperature. The following antibody were used: Mouse monoclonal anti-Flag (M2) peroxidase (HRP) (SigmaAldrich, Cat\#A8592, 1:1000), Mouse monoclonal anti-c-Myc-HRP (Roche Applied Science, Cat\#11814150001, 1:1500), Mouse monoclonal anti- $\beta$-actin (SigmaAldrich, Cat\# A3854, 1:3000). After washing with PBST, Immobilon Western Chemiluminescent HRP Substrate (Millipore) was used for secondary protein detection. The bands were visualized by chemical composition using ChemiDoc Touch (BIO-RAD). The uncropped and unprocessed scans of SDS-PAGE is provided in the source data file.

For IL-6 degradation assay, $2.5 \times 10^{5} 293 \mathrm{~T}$ cells were plated into 24 -well cell culture plate for 24 h. $0.06 \mathrm{pM} Z c 3 h 12 a$ or $Z c 3 h 12 c$ containing pLVX-vector were co-transfected with 0.01pM IL6-CDS or IL6-CDS-3UTR containing pWPI-plasmid into $293 \mathrm{~T}$ cell in the presence of PEI. After $48 \mathrm{~h}$, supernatants were collected for ELISA, and mRNAs were extracted for qPCR.

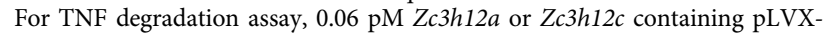
vector were co-transfected with $0.03 \mathrm{pM}$ pWPI-TNF-CDS or TNF-CDS-3UTR containing plasmid. After $48 \mathrm{~h}$, supernatants were collected for ELISA.

RNA extraction and quantitative PCR (qPCR). TRIzol reagent (Genstar, Cat\#P118-05) and chloroform were added to homogenize single cells, followed by RNA precipitation, washing and resuspension following the manufacturer's protocol. The extracted RNA was used for reverse transcription according to the manufacturer's protocol (HiScript III RT SuperMix for qPCR, Vazyme, R323-01). Quantitative RT-PCR analysis was performed with SYBR Select Master Mix (Genstar, Cat\#A301-10) using StepOne Plus (Life Sciences). All data were normalized to $\beta$-Actin expression. qPCR primer sequences are listed in Supplementary Table 1 .

ELISA. Samples were placed on pre-coated plates with anti-mouse TNFa (Cat \# 887324-88, Thermofisher), IL-6 (Catalog \# 88-7064-88 Thermofisher), IFNa (Cat\# \# BMS6027, Invitrogen), and performed according to manufacturer's instructions.

Statistical analysis. Statistical analysis was performed in GraphPad Prism 6 software. For reanalysis of human microarray samples, two-tailed Wilcoxon Matchedpair Signed Rank Test was used. For murine samples, comparisons between two groups were performed by two-tailed Student's $t$ test. For human PBMC samples, two-tailed Mann-Whitney test was used. Two human samples (one each from CD4 ${ }^{+}$ $\mathrm{T}$ and macrophages groups) were excluded as outliers, due to their Z-scores exceeding \pm 3 . A $P$ value of 0.05 or less was considered significant. The survive curves were calculated using Log-rank (Mantel-Cox) test.

Reporting summary. Further information on research design is available in the Nature Research Reporting Summary linked to this article.

\section{Data availability}

No novel datasets are generated in this study. Data that support the findings of this study are reanalysis of publicly available datasets. Patient biopsy datasets are available at Gene Expression Omnibus (GEO) repository (https://www.ncbi.nlm.nih.gov/gdsin) with accession codes of GDS3539; GDS4600; GSE53552. RNA-seq datasets are available at The Immunological Genome Project (ImmGen) (https://www.immgen.org) with the search term "Zc3h12c" (http://rstats.immgen.org/Skyline/skyline.html). SNP analysis is available at The Genotype-Tissue Expression (GTEx) (https://www.gtexportal.org/) with the search term of "rs4561177" (https://www.gtexportal.org/home/snp/ rs4561177). Source data are provided with this paper.

Received: 26 October 2020; Accepted: 16 June 2021; Published online: 02 July 2021

\section{References}

1. Medzhitov, R. Origin and physiological roles of inflammation. Nature 454, 428-435 (2008)

2. Hunter, C. A. \& Jones, S. A. IL-6 as a keystone cytokine in health and disease. Nat. Immunol. 16, 448-457 (2015).

3. Kalliolias, G. D. \& Ivashkiv, L. B. TNF biology, pathogenic mechanisms and emerging therapeutic strategies. Nat. Rev. Rheumatol. 12, 49-62 (2016).

4. Bradley, J. R. TNF-mediated inflammatory disease. J. Pathol. 214, 149-160 (2008).

5. Fishman, D. et al. The effect of novel polymorphisms in the interleukin-6 (IL6) gene on IL-6 transcription and plasma IL-6 levels, and an association with systemic-onset juvenile chronic arthritis. J. Clin. Investig. 102, 1369-1376 (1998).

6. Tanaka, Y. \& Martin, E. Mola, IL-6 targeting compared to TNF targeting in rheumatoid arthritis: studies of olokizumab, sarilumab and sirukumab. Ann. Rheum. Dis. 73, 1595-1597 (2014).

7. Turner, M. \& Diaz-Munoz, M. D. RNA-binding proteins control gene expression and cell fate in the immune system. Nat. Immunol. 19, 120-129 (2018).

8. Fu, M. \& Blackshear, P. J. RNA-binding proteins in immune regulation: a focus on CCCH zinc finger proteins. Nat. Rev. Immunol. 17, 130-143 (2017).

9. Carballo, E., Lai, W. S. \& Blackshear, P. J. Feedback inhibition of macrophage tumor necrosis factor-alpha production by tristetraprolin. Science 281, 1001-1005 (1998).

10. Vinuesa, C. G. et al. ubiquitin ligase family member required to repress follicular helper T cells and autoimmunity. Nature 435, 452-458 (2005).

11. Liang, J. et al. A novel CCCH-zinc finger protein family regulates proinflammatory activation of macrophages. J. Biol. Chem. 283, 6337-6346 (2008).

12. Leppek, K. et al. Roquin promotes constitutive mRNA decay via a conserved class of stem-loop recognition motifs. Cell 153, 869-881 (2013).

13. Huang, S. et al. Monocyte chemotactic protein-induced protein 1 and 4 form a complex but act independently in regulation of interleukin- 6 mRNA degradation. J. Biol. Chem. 290, 20782-20792 (2015).

14. Mino, T. et al. Regnase- 1 and roquin regulate a common element in inflammatory mRNAs by spatiotemporally distinct mechanisms. Cell 161, 1058-1073 (2015)

15. Matsushita, K. et al. Zc3h12a is an RNase essential for controlling immune responses by regulating mRNA decay. Nature 458, 1185-1190 (2009).

16. Linterman, M. A. et al. Roquin differentiates the specialized functions of duplicated $\mathrm{T}$ cell costimulatory receptor genes $\mathrm{CD} 28$ and ICOS. Immunity $\mathbf{3 0}$, 228-241 (2009).

17. Uehata, T. et al. Malt1-induced cleavage of regnase-1 in CD4(+) helper T cells regulates immune activation. Cell 153, 1036-1049 (2013).

18. Vogel, K. U. et al. Roquin paralogs 1 and 2 redundantly repress the Icos and Ox40 costimulator mRNAs and control follicular helper $\mathrm{T}$ cell differentiation. Immunity 38, 655-668 (2013).

19. Essig, K. et al. Roquin suppresses the PI3K-mTOR signaling pathway to inhibit $\mathrm{T}$ helper cell differentiation and conversion of Treg to Tfr Cells. Immunity 47, 1067-1082.e1012 (2017).

20. Garg, A. V. et al. MCPIP1 endoribonuclease activity negatively regulates interleukin-17-mediated signaling and inflammation. Immunity 43, 475-487 (2015).

21. Taylor, G. A. et al. A pathogenetic role for TNF alpha in the syndrome of cachexia, arthritis, and autoimmunity resulting from tristetraprolin (TTP) deficiency. Immunity 4, 445-454 (1996).

22. Liang, J., Song, W., Tromp, G., Kolattukudy, P. E. \& Fu, M. Genome-wide survey and expression profiling of CCCH-zinc finger family reveals a functional module in macrophage activation. PLoS ONE 3, e2880 (2008)

23. Minagawa, K. et al. Posttranscriptional modulation of cytokine production in $\mathrm{T}$ cells for the regulation of excessive inflammation by TFL. J. Immunol. 192, 1512-1524 (2014)

24. von Gamm, M. et al. Immune homeostasis and regulation of the interferon pathway require myeloid-derived Regnase-3. J Exp Med 216, 1700-1723 (2019). 
25. Tsoi, L. C. et al. Identification of 15 new psoriasis susceptibility loci highlights the role of innate immunity. Nat. Genet. 44, 1341-1348 (2012).

26. Perera, G. K., Di Meglio, P. \& Nestle, F. O. Psoriasis. Annu Rev. Pathol. 7, 385-422 (2012).

27. Lowes, M. A., Suarez-Farinas, M. \& Krueger, J. G. Immunology of psoriasis. Annu Rev. Immunol. 32, 227-255 (2014).

28. Dainichi, T. et al. The epithelial immune microenvironment (EIME) in atopic dermatitis and psoriasis. Nat. Immunol. 19, 1286-1298 (2018).

29. Prinz, I., Sandrock, I. \& Mrowietz, U. Interleukin-17 cytokines: effectors and targets in psoriasis-A breakthrough in understanding and treatment. J. Exp. Med. 217, e20191397 (2020)

30. Yao, Y. et al. Type I interferon: potential therapeutic target for psoriasis? PLoS ONE 3, e2737 (2008).

31. Suarez-Farinas, M. et al. Expanding the psoriasis disease profile: interrogation of the skin and serum of patients with moderate-to-severe psoriasis. J. Investig. Dermatol. 132, 2552-2564 (2012).

32. Russell, C. B. et al. Gene expression profiles normalized in psoriatic skin by treatment with brodalumab, a human anti-IL-17 receptor monoclonal antibody. J. Immunol. 192, 3828-3836 (2014).

33. Guiducci, C. et al. Properties regulating the nature of the plasmacytoid dendritic cell response to Toll-like receptor 9 activation. J. Exp. Med. 203, 1999-2008 (2006).

34. Caton, M. L., Smith-Raska, M. R. \& Reizis, B. Notch-RBP-J signaling controls the homeostasis of CD8- dendritic cells in the spleen. J. Exp. Med. 204, 1653-1664 (2007).

35. Clausen, B. E., Burkhardt, C., Reith, W., Renkawitz, R. \& Forster, I Conditional gene targeting in macrophages and granulocytes using LysMcre mice. Transgenic Res. 8, 265-277 (1999).

36. Armstrong, A. W. \& Read, C. Pathophysiology, clinical presentation, and treatment of psoriasis: a review. JAMA 323, 1945-1960 (2020).

37. Murray, P. J. Macrophage polarization. Annu Rev. Physiol. 79, 541-566 (2017).

38. Gordon, S. \& Martinez, F. O. Alternative activation of macrophages: mechanism and functions. Immunity 32, 593-604 (2010).

39. Colonna, M., Trinchieri, G. \& Liu, Y. J. Plasmacytoid dendritic cells in immunity. Nat. Immunol. 5, 1219-1226 (2004).

40. Cervantes-Barragan, L. et al. Plasmacytoid dendritic cells control T-cell response to chronic viral infection. Proc. Natl Acad. Sci. U.S.A. 109, 3012-3017 (2012).

41. Nestle, F. O. et al. Plasmacytoid predendritic cells initiate psoriasis through interferon-alpha production. J. Exp. Med. 202, 135-143 (2005).

42. Stratis, A. et al. Pathogenic role for skin macrophages in a mouse model of keratinocyte-induced psoriasis-like skin inflammation. J. Clin. Investig. 116, 2094-2104 (2006).

43. Wang, $\mathrm{H}$. et al. Activated macrophages are essential in a murine model for $\mathrm{T}$ cell-mediated chronic psoriasiform skin inflammation. J. Clin. Investig. 116, 2105-2114 (2006).

44. Lande, R. et al. Plasmacytoid dendritic cells sense self-DNA coupled with antimicrobial peptide. Nature 449, 564-569 (2007)

45. Ganguly, D. et al. Self-RNA-antimicrobial peptide complexes activate human dendritic cells through TLR7 and TLR8. J. Exp. Med. 206, 1983-1994 (2009).

46. Glitzner, E. et al. Specific roles for dendritic cell subsets during initiation and progression of psoriasis. EMBO Mol. Med. 6, 1312-1327 (2014).

47. Wohn, C. et al. Langerin(neg) conventional dendritic cells produce IL-23 to drive psoriatic plaque formation in mice. Proc. Natl Acad. Sci. U.S.A. 110, 10723-10728 (2013).

48. van der Fits, L. et al. Imiquimod-induced psoriasis-like skin inflammation in mice is mediated via the IL-23/IL-17 axis. J. Immunol. 182, 5836-5845 (2009).

49. Albanesi, C. et al. Chemerin expression marks early psoriatic skin lesions and correlates with plasmacytoid dendritic cell recruitment. J. Exp. Med. 206, 249-258 (2009).

50. Reizis, B. Plasmacytoid dendritic cells: development, regulation, and function. Immunity 50, 37-50 (2019).

51. Chen, Y. L. et al. Re-evaluation of human BDCA-2+ DC during acute sterile skin inflammation. J. Exp. Med 217, e20190811 (2020).

52. Leylek, R. et al. Integrated cross-species analysis identifies a conserved transitional dendritic cell population. Cell Rep. 29, 3736-3750.e3738 (2019).

53. Honigsmann, H. Phototherapy for psoriasis. Clin. Exp. Dermatol. 26, 343-350 (2001).

54. Tamoutounour, S. et al. Origins and functional specialization of macrophages and of conventional and monocyte-derived dendritic cells in mouse skin. Immunity 39, 925-938 (2013).

55. Hedrick, M. N. et al. CCR6 is required for IL-23-induced psoriasis-like inflammation in mice. J. Clin. Investig. 119, 2317-2329 (2009).

56. Tilg, H., Trehu, E., Atkins, M. B., Dinarello, C. A., Mier, J. W. Interleukin-6 (IL-6) as an anti-inflammatory cytokine: induction of circulating IL-1 receptor antagonist and soluble tumor necrosis factor receptor p55. Blood 83, 113-118 (1994).

57. Sharif, M. N. et al. Twist mediates suppression of inflammation by type I IFNs and Axl. J. Exp. Med. 203, 1891-1901 (2006).
58. Huang, S. et al. MCPIP1 negatively regulates toll-like receptor 4 signaling and protects mice from LPS-induced septic shock. Cell Signal 25, 1228-1234 (2013).

59. Werner, S. L. et al. Encoding NF-kappaB temporal control in response to TNF: distinct roles for the negative regulators IkappaBalpha and A20. Genes Dev. 22, 2093-2101 (2008).

60. Garbers, C., Aparicio-Siegmund, S. \& Rose-John, S. The IL-6/gp130/ STAT3 signaling axis: recent advances towards specific inhibition. Curr. Opin. Immunol. 34, 75-82 (2015)

61. Grossman, R. M. et al. Interleukin 6 is expressed in high levels in psoriatic skin and stimulates proliferation of cultured human keratinocytes. Proc. Natl Acad. Sci. U.S.A. 86, 6367-6371 (1989).

62. Fritz, Y. et al. Induction of alternative proinflammatory cytokines accounts for sustained psoriasiform skin inflammation in IL-17C+IL-6KO mice. J. Investig. Dermatol. 137, 696-705 (2017).

63. Wendling, D., Letho-Gyselinck, H., Guillot, X. \& Prati, C. Psoriasis onset with tocilizumab treatment for rheumatoid arthritis. J. Rheumatol. 39, 657 (2012).

64. Lindroos, J. et al. IL-23-mediated epidermal hyperplasia is dependent on IL-6. J. Investig. Dermatol 131, 1110-1118 (2011).

65. Gregorio, J. et al. Plasmacytoid dendritic cells sense skin injury and promote wound healing through type I interferons. J. Exp. Med. 207, 2921-2930 (2010).

66. Guiducci, C. et al. Autoimmune skin inflammation is dependent on plasmacytoid dendritic cell activation by nucleic acids via TLR7 and TLR9. J. Exp. Med. 207, 2931-2942 (2010).

67. Kimball, A. S. et al. Gallagher, the histone methyltransferase setdb2 modulates macrophage phenotype and uric acid production in diabetic wound repair. Immunity 51, 258-271.e255. (2019).

68. Yamamoto, M. et al. Regulation of Toll/IL-1-receptor-mediated gene expression by the inducible nuclear protein IkappaBzeta. Nature 430, 218-222 (2004).

69. Tartey, S. et al. Akirin2 is critical for inducing inflammatory genes by bridging IkappaB-zeta and the SWI/SNF complex. EMBO J. 33, 2332-2348 (2014).

70. Motoyama, M., Yamazaki, S., Eto-Kimura, A., Takeshige, K. \& Muta, T. Positive and negative regulation of nuclear factor-kappaB-mediated transcription by IkappaB-zeta, an inducible nuclear protein. J. Biol. Chem. 280, 7444-7451 (2005).

\section{Acknowledgements}

We would like to thank Drs. Ananda W. Goldrath for LCMV-Armstrong/CL13, VSV-OVA, and LM-OVA, Juncao Cai for luciferase reporter plasmids and cell lines, and Cui Jun for Western blotting experiments. We are grateful for the critical reading of the paper by Drs. Xiaojun Xia and Vanja Sisirak. We thank Dr. Weibin Cai for technical assistance at the animal facilities. This research is supported by the National Key R\&D Program of China (2018YFA0508300) to C.Y.Y., the National Natural Science Foundation of China (81771772) to C.Y.Y., the Guangdong Innovative and Entrepreneurial Research Team Program (2016ZT06S638) to C.Y.Y., and the 111 Project (Grant No B12003) to C.Y.Y.; National Natural Science Foundation of China (82071820 and 81601404) to J.Z.; Ramanujan Fellowship to D.G; Senior Research Fellowship from University Grants Commission to O.R.

\section{Author contributions}

B.L. designed, performed experiments, analyzed the data, and wrote the paper. J.H., A.A., J.L., S.A., L.W., P.C., X.L., H.N., and J.W. performed experiments. D.G. designed and supervised experiments involving human samples. O.R. performed and analyzed experiments with human samples. J.Z. designed and supervised experiments, oversaw protocol logistics for human samples, interpreted the data, wrote the paper, and contributed to funding. C.Y.Y. conceived of the study, designed experiments, supervised the entire study, oversaw protocol logistics, analyzed and interpreted the data, and wrote the paper.

\section{Competing interests}

The authors declare no competing interests.

\section{Additional information}

Supplementary information The online version contains supplementary material available at https://doi.org/10.1038/s41467-021-24352-w.

Correspondence and requests for materials should be addressed to J.Z. or C.Y.Y.

Peer review information Nature Communications thanks Mingui Fu and Peter Wolf for their contribution to the peer review of this work.

Reprints and permission information is available at http://www.nature.com/reprints

Publisher's note Springer Nature remains neutral with regard to jurisdictional claims in published maps and institutional affiliations. 
(c) (i) Open Access This article is licensed under a Creative Commons Attribution 4.0 International License, which permits use, sharing, adaptation, distribution and reproduction in any medium or format, as long as you give appropriate credit to the original author(s) and the source, provide a link to the Creative Commons license, and indicate if changes were made. The images or other third party material in this article are included in the article's Creative Commons license, unless indicated otherwise in a credit line to the material. If material is not included in the article's Creative Commons license and your intended use is not permitted by statutory regulation or exceeds the permitted use, you will need to obtain permission directly from the copyright holder. To view a copy of this license, visit http://creativecommons.org/ licenses/by/4.0/.

(C) The Author(s) 2021 